Annals of Pure and Applied Mathematics

Vol. 15, No. 2, 2017, 357-373

ISSN: 2279-087X (P), 2279-0888(online)

Published on 11 December 2017

www.researchmathsci.org

DOI: http://dx.doi.org/10.22457/apam.v15n2a22

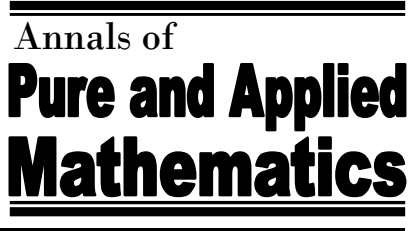

\title{
Mean Time to Recruitment for a Multigrade Manpower System with Two Sources of Depletion when Wastages form an order Statistics and the Breakdown Threshold Distribution Follows SCBZ Property
}

\author{
K.Srividhya ${ }^{1}$ and S.Sendhamizh Selvi ${ }^{2}$ \\ ${ }^{1}$ Department of Mathematics, National College, Trichy-620001 \\ Tamilnadu, India. Email: drsrimano@gmail.com \\ ${ }^{2} \mathrm{PG}$ and Research Department of Mathematics, Government Arts College \\ Trichy-620022, Tamilnadu, India. email: sendhamizhs@yahoo.co.in
}

Received 28 November 2017; accepted 8 December 2017

Abstract. In this paper a multi graded organization in which depletion of manpower occur due to policy and transfer decisions is considered when the breakdown threshold distribution follows SCBZ property. Mean time to recruitment is obtained by using anunivariate CUM policy of recruitment (ie) "The organization survives iff atleast $r(1 \leq r \leq n)$ out of $\mathrm{n}$ grades survives in the sense that threshold crossing has not take place in these grades" when wastages form an order statistics. The influence of the nodal parameter on the system characteristics is studied and relevant conclusions are presented

Keywords: Loss of man hours, Policy decision, Transfer decision, Order statistics, SCBZ property.

AMS Mathematics Subject Classification (2010): 00A71

\section{Introduction}

Exits of personal which is in other words known as wastage, is an important aspects in the study of manpower planning. Many models have been discussed using different types of wastages and also different types of distribution for the loss of man powers, the thresholds and inter decision times. Such models are seen in $[1,2]$. In $[3,4,5,6]$ the authors have obtained the mean time to recruitment in a two grade manpower system based on order statistics by assuming different distribution for thresholds. In [8] for a two grade manpower system with two types of decisions when the wastages form a geometric process is obtained. The problem of time to recruitment is studied by several authors for the organizations consisting of single grade/two grade/ three grades .More specifically for a two grade system, in all the earlier work, the threshold for the organization is minimum or maximum or sum of the thresholds for the loss of manpower in each grades, no attempt has been made so far to design a comprehensive recruitment policy for a system with two or three grades. In $[10,11,12]$ a new design for a comprehensive univariate CUM recruitment policy of manpower system is used with $\mathrm{n}$ grades in order to bring results proved independently for maximum, minimum model as a special case. In all 


\section{K.Srividhya and S.Sendhamizh Selvi}

previous work, the problem of time to recruitment is studied for only an organization consisting of atmost three grades. $\operatorname{In}[11,12]$ author has worked on this comprehensive univariate policy when wastages form ordinary renewal process and inter decision time form geometric and order statistics.Here an attempt is made for loss of manpower due to policy and transfer decision with high and low attrition rate for inter policy decision.

\section{Model description and assumptions}

An organization having n-grades in which decisions are taken at random epochs $(0, \infty)$ is considered. At every policy decision epoch a random number of person quit the organization and at every transfer decision epoch a random number of persons are transferred. It is assumed that the loss of manpower is linear and cumulative. The loss of manpower process, process of inter policy and inter transfer decision times are statistically independent. The loss of man power follows order statistics, inter policy decision times are hyper exponential and inter transfer decision times are exponential. The thresholds for the n-grades are independent and identically distributed random variables following SCBZ property with same parameter. Univariate CUM policy of recruitment "The organization survives iff at least $r(1 \leq r \leq n)$ out of $\mathrm{n}$ grades survives in the sense that threshold crossing has not take place in these grades".

$x_{i}$ : Continuous random variable denoting the amount of depletion of manpower caused due to the $\mathrm{i}^{\text {th }}$ policy decision in organization. $t_{i}$ :Time of occurrence of the $\mathrm{i}^{\text {th }}$ decision. $\bar{x}_{m 1}$ :Cumulative loss of manpower due to the first $m 1$ policy decisions in the Organization. $y_{j}$ : Continuous random variable denoting the amount of depletion of manpowers caused due to the ${ }^{\text {th }}$ transfer decision in organization.

$\bar{y}_{n 1}$ : Cumulative loss of manpower due to the first $n 1$ transfer decisions in the Organization.

$W_{U}($.$) :The distribution function of inter policy decision times with hyper exponential$ i.i.d random variable.

$W_{V}($.$) :The distribution function of inter transfer decision times with exponential i.i.d$ random variable.

$W_{U}^{m 1}():. m 1$ fold convolution of $W_{U}$ with itself.

$W_{V}^{n 1}():. n 1$ fold convolution of $W_{V}$ with itself.

$\bar{x}_{m 1}+\bar{y}_{n 1}$ : The cumulative loss of manpower due to $m 1$ policy decisions and $n 1$ transfer decision.

$\bar{W}_{\bar{x}_{m 1}+\bar{y}_{n 1}}$ : Distribution function of cumulative loss of manpower due to $m 1$ policy decisions and $n 1$ transfer decision.

T: Time to recruitment.

$E(T)$ : Mean time to recruitment.

$N_{P}(T)$ : Number of policy decisions at time $\mathrm{T}$.

$N_{\text {Trans }}(T)$ : Number of transfer decisions at time $\mathrm{T}$.

\section{Main results}

From renewal theory, the survival function of $\mathrm{T}$ is

$$
\left.P(T>t)=P\left(\bar{X}_{N_{P}}(T)+\bar{Y}_{N_{\text {Trans }}}(T)\right)<Z\right)
$$

Conditioning upon $N_{P}(T)$ and $N_{\text {Trans }}(T)$ and using law of total probability

$$
P(T>t)=\sum_{m 1=0}^{\infty} P\left(N_{P}(T)=m 1\right) \sum_{n 1}^{\infty} P\left(N_{\text {Trans }}(T)=n 1\right) P\left(\bar{x}_{m 1}+\bar{y}_{n 1} \leq Z\right)(2)
$$


Mean Time to Recruitment for a Multigrade Manpower System with Two Sources ...

As $\left\{N_{P}(T)\right\}$ and $\left\{N_{\text {Trans }}(T)\right\}$ are two independent ordinary renewal process by hypothesis invoking the

$$
\begin{aligned}
& \text { result. }\left(N_{P}(T)=m_{1}\right)=W_{U}^{m 1}(t)-W_{U}^{m 1+1}(t) \operatorname{and} P\left(N_{\text {Trans }}(T)=n_{1}\right)=W_{V}^{n 1}(t)- \\
& W_{V}^{n 1+1}(t) \\
& \quad P(T>t)=\sum_{m 1=0}^{\infty}\left[W_{U}^{m 1}(t)-W_{U}^{m 1+1}(t)\right] \sum_{n 1}^{\infty}\left[W_{V}^{n 1}(t)-W_{V}^{n 1+1}(t)\right] P\left(\bar{x}_{m 1}+\bar{y}_{n 1} \leq Z\right)(3) \\
& \text { where } W_{U}^{0}(t)=W_{V}^{0}(t)=1
\end{aligned}
$$

Let $z_{j}, j=1,2, \ldots n$ follows SCBZ property with same parameter

$H\left(z_{j}\right)=\left\{\begin{array}{c}1-e^{-\theta_{1} z_{j}} \quad z_{j} \leq \tau_{0} \\ 1-e^{-\theta_{1} z_{j}} e^{-\theta_{2}\left(z_{j}-\tau_{0}\right)} \tau_{0} \leq z_{j}\end{array} j=1, \ldots n\right.$ for fixed $\tau=\tau_{0}$

Assuming the truncation level, itself a random variable such that $\tau$ follows exponential with parameter $\theta$, we have by law of total probability,

$$
H(z)=1-p e^{-\left(\theta+\theta_{1}\right) z}-q e^{-\theta_{2} z}
$$

where $p=\left(\frac{\theta_{1}-\theta_{2}}{\theta+\theta_{1}-\theta_{2}}\right)$ and $q=\left(\frac{\theta}{\theta+\theta_{1}-\theta_{2}}\right)$ with $\mathrm{p}+\mathrm{q}=1(5)$

Since $\mathrm{Z}$ is independent of $\bar{x}_{m 1}$ and $\bar{y}_{n 1}$, by hypothesis conditioning upon $\mathrm{z}$ and using law of total probability

where

$$
P\left(\bar{x}_{m 1}+\bar{y}_{n 1} \leq \mathrm{Z}\right)=\int_{0}^{\infty} P\left(\bar{x}_{m 1}+\bar{y}_{n 1}<z\right) h(z) d z
$$

$$
\begin{aligned}
& h(z)=\sum_{i=r}^{n} n C_{i}\left\{\left\{p^{i} i\left(\theta+\theta_{1}\right) e^{-i\left(\theta+\theta_{1}\right) z}+\cdots q^{i} i \theta_{2} e^{-i\left(\theta_{2}\right) z}\right\}\right. \\
& -(n-i) C_{1}\left\{p^{i+1}(i+1)\left(\theta+\theta_{1}\right) e^{-(i+1)\left(\theta+\theta_{1}\right) z}+\cdots q^{i+1}(i+1)\left(\theta_{2}\right) e^{-(i+1)\left(\theta_{2}\right) z}\right\} \\
& \left.\cdots(-1)^{n-i}\left\{p^{n} n\left(\theta+\theta_{1}\right) e^{-n\left(\theta+\theta_{1}\right) z}+\cdots q^{n} n \theta_{2} e^{-n\left(\theta_{2}\right) z}\right\}\right\} \\
& P\left(\bar{x}_{m 1}+\bar{y}_{n 1} \leq \mathrm{Z}\right) \\
& =\int_{0}^{\infty} P\left(\bar{x}_{m 1}+\bar{y}_{n 1}<z\right) \\
& \times \sum_{i=r}^{n} n C_{i}\left\{\begin{array}{c}
\left\{(n-i) C_{1}\left\{p^{i+1}(i+1)\left(\theta+\theta_{1}\right) e^{-(i+1)\left(\theta+\theta_{1}\right) z}+\cdots q^{i+1}(i \quad+1)\left(\theta_{2}\right) e^{-(i+1)\left(\theta_{2}\right) z}\right\}+\right. \\
\cdots(-1)^{n-i}\left\{p^{n} n\left(\theta+\theta_{1}\right) e^{-n\left(\theta+\theta_{1}\right) z}+\cdots q^{n} n \theta_{2} e^{-n\left(\theta_{2}\right) z}\right\}
\end{array}\right\} d z
\end{aligned}
$$

Substituting equation (8) in (3)

$$
\begin{aligned}
& P(T>t)=\sum_{m 1=0}^{\infty}\left[W_{U}{ }^{m 1}(t)-W_{U}{ }^{m 1+1}(t)\right] \sum_{n 1}^{\infty}\left[{W_{V}}^{n 1}(t)-W_{V}{ }^{n 1+1}(t)\right] \\
& \int_{0}^{\infty} P\left(\bar{x}_{m 1}+\bar{y}_{n 1}<z\right) \\
& \times \sum_{i=r}^{n} n C_{i}\left\{\begin{array}{c}
\left\{p^{i} i\left(\theta+\theta_{1}\right) e^{-i\left(\theta+\theta_{1}\right) z}+\cdots q^{i} i \theta_{2} e^{-i\left(\theta_{2}\right) z}\right\} \\
-(n-i) C_{1}\left\{p^{i+1}(i+1)\left(\theta+\theta_{1}\right) e^{-(i+1)\left(\theta+\theta_{1}\right) z}+\cdots q^{i+1}(i+1)\left(\theta_{2}\right) e^{-(i+1)\left(\theta_{2}\right) z}\right\}+ \\
\cdots(-1)^{n-i}\left\{p^{n} n\left(\theta+\theta_{1}\right) e^{-n\left(\theta+\theta_{1}\right) z}+\cdots q^{n} n \theta_{2} e^{-n\left(\theta_{2}\right) z}\right\}
\end{array}\right\} d z
\end{aligned}
$$


K.Srividhya and S.Sendhamizh Selvi

$$
\begin{aligned}
& P(T>t) \\
& =\sum_{m 1=0}^{\infty}\left[W_{U}^{m 1}(t)-W_{U}^{m 1+1}(t)\right] \sum_{n 1}^{\infty}\left[W_{V}^{n 1}(t)-W_{V}^{n 1+1}(t)\right] \\
& \times \sum_{i=r}^{n} n C_{i}\left\{\begin{array}{c}
\left\{p^{i} \bar{w}_{\bar{x}_{m 1}+\bar{y}_{n 1}}\left(i\left(\theta+\theta_{1}\right)\right)+\cdots+q^{i} \bar{w}_{\bar{x}_{m 1}+\bar{y}_{n 1}}\left(i\left(\theta_{2}\right)\right)\right\}- \\
(n-i) C_{1}\left\{p^{i+1} \bar{w}_{\bar{x}_{m 1}+\bar{y}_{n 1}}\left((i+1)\left(\theta+\theta_{1}\right)\right)+\cdots q^{i+1} \bar{w}_{\bar{x}_{m 1}+\bar{y}_{n 1}}\left((i+1) \theta_{2}\right)\right\}+ \\
\cdots(-1)^{n-i}\left\{p^{n} \bar{w}_{\bar{x}_{m 1}+\bar{y}_{n 1}}\left(n\left(\theta+\theta_{1}\right)+\cdots+q^{n} \bar{w}_{\bar{x}_{m 1}+\bar{y}_{n 1}}\left(n\left(\theta_{2}\right)\right)\right\}\right.
\end{array}\right\}
\end{aligned}
$$

where $\bar{w}_{\bar{x}_{m 1}+\bar{y}_{n 1}}(\theta)=\left\{\bar{w}_{\bar{x}_{i}}(\theta)\right\}^{m 1}\left\{\bar{w}_{\bar{y}_{i}}(\theta)\right\}^{n 1}(11)$

Using (11) in (10)

$$
P(T>t)=\sum_{i=r}^{n} n C_{i}\left\{\begin{array}{c}
\left\{p^{i} D_{i\left(\theta+\theta_{1}\right)}(t)+\cdots+q^{i} D_{i\left(\theta_{2}\right)}(t)\right\}- \\
(n-i) C_{1}\left\{p^{i+1} D_{(i+1)\left(\theta+\theta_{1}\right)}(t)+\cdots+q^{i+1} D_{(i+1)\left(\theta_{2}\right)}(t)\right\}+. . \\
(-1)^{n-i}\left\{p^{n} D_{n\left(\theta+\theta_{1}\right)}(t)+\cdots+q^{n} D_{n\left(\theta_{2}\right)}(t)\right\}
\end{array}\right\}
$$

where

$$
D_{\theta}(t)=\sum_{m 1=0}^{\infty}\left[W_{U}{ }^{m 1}(t)-W_{U}^{m 1+1}(t)\right]\left\{\bar{w}_{\bar{x}_{i}}(\theta)\right\}^{m 1} \sum_{n 1}^{\infty}\left[W_{V}{ }^{n 1}(t)-W_{V}{ }^{n 1+1}(t)\right]\left\{\bar{w}_{\bar{y}_{i}}(\theta)\right\}^{n 1}
$$

Expanding and simplifying the equation (13)

$$
\begin{aligned}
& D_{\theta}(t)=\left\{1-\left[1-\bar{W}_{\bar{x}_{i}}(\theta)\right] \sum_{m 1=0}^{\infty} W_{U}^{m 1}(t)\left\{\bar{w}_{\bar{x}_{i}}(\theta)\right\}^{m 1-1}\right\} \\
& \quad \times\left\{1-\left[1-\bar{W}_{\bar{y}_{i}}(\theta)\right] \sum_{n 1=0}^{\infty} W_{V}^{n 1}(t)\left\{\bar{w}_{\bar{y}_{i}}(\theta)\right\}^{n 1-1}\right\} \\
& G_{\theta}(t)=1-D_{\theta}(t) \\
& \quad=\left\{\left[1-\bar{W}_{\bar{x}_{i}}(\theta)\right] \sum_{m 1=0}^{\infty} W_{U}^{m 1}(t)\left\{\bar{w}_{\bar{x}_{i}}(\theta)\right\}^{m 1-1}\right\}+\left\{\left[1-\bar{W}_{\bar{y}_{i}}(\theta)\right] \sum_{n 1=0}^{\infty} W_{V}{ }^{n 1}(t)\left\{\bar{w}_{\bar{y}_{i}}(\theta)\right\}^{n 1-1}\right\} \\
& -\left\{\left[1-\bar{W}_{\bar{x}_{i}}(\theta)\right] \sum_{m 1=0}^{\infty} W_{U}^{m 1}(t)\left\{\bar{w}_{\bar{x}_{i}}(\theta)\right\}^{m 1-1}\right\}\left\{\left[1-\bar{W}_{\bar{y}_{i}}(\theta)\right] \sum_{n 1=0}^{\infty} W_{V}{ }^{n 1}(t)\left\{\bar{w}_{\bar{y}_{i}}(\theta)\right\}^{n 1-1}\right\} \\
& g_{\theta}(t)=\frac{d}{d t}\left(G_{\theta}(t)\right) \\
& =\left\{\left[1-\bar{w}_{\bar{x}_{i}}(\theta)\right] \sum_{m 1=0}^{\infty} w_{U}^{m 1}(t)\left\{\bar{w}_{\bar{x}_{i}}(\theta)\right\}^{m 1-1}\right\}+\left\{\left[1-\bar{w}_{\bar{y}_{i}}(\theta)\right] \sum_{n 1=0}^{\infty} w_{V}{ }^{n 1}(t)\left\{\bar{w}_{\bar{y}_{i}}(\theta)\right\}^{n 1-1}\right\} \\
& \quad-\left\{\left[1-\bar{w}_{\bar{x}_{i}}(\theta)\right] \sum_{m 1=0}^{\infty} W_{U}{ }^{m 1}(t)\left\{\bar{w}_{\bar{x}_{i}}(\theta)\right\}^{m 1-1}\right\}\left\{\left[1-\bar{w}_{\bar{y}_{i}}(\theta)\right] \sum_{n 1=0}^{\infty} w_{V}{ }^{n 1}(t)\left\{\bar{w}_{\bar{y}_{i}}(\theta)\right\}^{n 1-1}\right\} \\
& -\left\{\left[1-\bar{w}_{\bar{x}_{i}}(\theta)\right] \sum_{m 1=0}^{\infty} w_{U}^{m 1}(t)\left\{\bar{w}_{\bar{x}_{i}}(\theta)\right\}^{m 1-1}\right\}\left\{\left[1-\bar{w}_{\bar{y}_{i}}(\theta)\right] \sum_{n 1=0}^{\infty} W_{V}^{n 1}(t)\left\{\bar{w}_{\bar{y}_{i}}(\theta)\right\}^{n 1-1}\right\}
\end{aligned}
$$

Since $\bar{w}_{V}(t)$ is exponential with parameter $\mu_{2}, \bar{w}_{V}{ }^{n 1}(t)$ is a gamma distribution with parameter $\mu_{2}, n 1$. Therefore, 
Mean Time to Recruitment for a Multigrade Manpower System with Two Sources ...

$$
\begin{gathered}
{\left[1-w_{\bar{y}_{i}}(\theta)\right] \sum_{n 1=0}^{\infty} W_{V}^{n 1}(t)\left\{\bar{w}_{\bar{y}_{i}}(\theta)\right\}^{n 1-1}=\mu_{2}\left[1-\bar{w}_{\bar{y}_{i}}(\theta)\right]\left[\frac{e^{-\mu_{2} u\left[1-\bar{w}_{\bar{y}_{i}}(\theta)\right]}}{\mu_{2}\left[1-\bar{w}_{\bar{y}_{i}}(\theta)\right]}\right]_{0}^{t}} \\
=1-e^{-\mu_{2}\left[1-\bar{w}_{\bar{y}_{i}}(\theta)\right] t}
\end{gathered}
$$

Therefore

$$
\left[1-\bar{w}_{\bar{y}_{i}}(\theta)\right] \sum_{n 1=0}^{\infty} w_{V}^{n 1}(t)\left\{\bar{w}_{\bar{y}_{i}}(\theta)\right\}^{n 1-1}=\mu_{2}\left[1-\bar{w}_{\bar{y}_{i}}(\theta)\right] e^{-\mu_{2}\left[1-\bar{w}_{\bar{y}_{i}}(\theta)\right] t}
$$

Substituting equation (16) and (17) in equation (15)

Taking Laplace Steljies transform on both side

$$
\begin{aligned}
g_{\theta}(t)=\mu_{2}\left[1-\bar{w}_{\bar{y}_{i}}(\theta)\right] e^{-\mu_{2}\left[1-\bar{w}_{\bar{y}_{i}}(\theta)\right] t}-\left\{\left[1-\bar{w}_{\bar{x}_{i}}(\theta)\right] \sum_{m 1=0}^{\infty} W_{U}^{m 1}(t)\left\{\bar{w}_{\bar{x}_{i}}(\theta)\right\}^{m 1-1}\right\} \\
\times\left\{\mu_{2}\left[1-\bar{w}_{\bar{y}_{i}}(\theta)\right]\right\} e^{-\mu_{2}\left[1-\bar{w}_{\bar{y}_{i}}(\theta)\right] t} \\
+\left\{\left[1-\bar{w}_{\bar{x}_{i}}(\theta)\right] \sum_{m=0}^{\infty} w_{U}{ }^{m 1}(t)\left\{\bar{w}_{\bar{x}_{i}}(\theta)\right\}^{m 1-1}\right\}\left[e^{-\mu_{2}\left[1-\bar{w}_{\bar{y}_{i}}(\theta)\right] t}\right]
\end{aligned}
$$

$\bar{g}_{\theta}(s)=\frac{\mu_{2}\left[1-\bar{w}_{\bar{y}_{i}}(\theta)\right]}{s+\mu_{2}\left[1-\bar{w}_{\bar{y}_{i}}(\theta)\right]}$

$$
\begin{aligned}
&\left\{\mu_{2}\left[1-\bar{w}_{\bar{y}_{i}}(\theta)\right]\left[1-\bar{w}_{\bar{x}_{i}}(\theta)\right] \sum_{m 1=0}^{\infty} \frac{\left[\bar{w}_{U}\left(s+\mu_{2}\left[1-\bar{w}_{\bar{y}_{i}}(\theta)\right]\right)\right]^{m 1}}{s+\mu_{2}\left[1-\bar{w}_{\bar{y}_{i}}(\theta)\right]}\left\{\bar{w}_{\bar{x}_{i}}(\theta)\right\}^{m 1-1}\right\} \\
&+\left\{\left[1-\bar{w}_{\bar{x}_{i}}(\theta)\right] \sum_{m 1=0}^{\infty}\left[\bar{w}_{U}\left(s+\mu_{2}\left[1-\bar{w}_{\bar{y}_{i}}(\theta)\right]\right)\right]^{m 1}\left\{\bar{w}_{\bar{x}_{i}}(\theta)\right\}^{m 1-1}\right\} \\
& \bar{g}_{\theta}(s)=\frac{\mu_{2}\left[1-\bar{w}_{\bar{y}_{i}}(\theta)\right]}{s+\mu_{2}\left[1-\bar{w}_{\bar{y}_{i}}(\theta)\right]}-\mu_{2}\left[1-\bar{w}_{\bar{y}_{i}}(\theta)\right]\left[1-\bar{w}_{\bar{x}_{i}}(\theta)\right] A(s)+\left[1-\bar{w}_{\bar{x}_{i}}(\theta)\right] B(s)(19)
\end{aligned}
$$

where

$$
\left.\begin{array}{c}
A(s)=\frac{\left[\bar{w}_{U}\left(s+\mu_{2}\left[1-\bar{w}_{\bar{y}_{i}}(\theta)\right]\right)\right]}{\left[s+\mu_{2}\left[1-\bar{w}_{\bar{y}_{i}}(\theta)\right]\right]\left[1-\left[\bar{w}_{U}\left(s+\mu_{2}\left[1-\bar{w}_{\bar{y}_{i}}(\theta)\right]\right)\left\{\bar{w}_{\bar{x}_{i}}(\theta)\right\}\right]\right]} \\
B(s)=\frac{\left[\bar{w}_{U}\left(s+\mu_{2}\left[1-\bar{w}_{\bar{y}_{i}}(\theta)\right]\right)\right]}{1-\left[\bar{w}_{U}\left(s+\mu_{2}\left[1-\bar{w}_{\bar{y}_{i}}(\theta)\right]\right)\left\{\bar{w}_{\bar{x}_{i}}(\theta)\right\}\right]}
\end{array}\right\}
$$

From equation (20)

$$
\begin{gathered}
\frac{d}{d s}(A(s)) \\
\left.=\frac{\left\{\begin{array}{c}
\left.\left\{s+\mu_{2}\left[1-\bar{w}_{\bar{y}_{i}}(\theta)\right]\right]\left[1-\left[\bar{w}_{U}\left(s+\mu_{2}\left[1-\bar{w}_{\bar{y}_{i}}(\theta)\right]\right)\left\{\bar{w}_{\bar{x}_{i}}(\theta)\right\}\right]\right]\left[\bar{w}_{U}{ }^{\prime}\left(s+\mu_{2}\left[1-\bar{w}_{\bar{y}_{i}}(\theta)\right]\right)\right]\right\}- \\
{\left[\bar{w}_{U}\left(s+\mu_{2}\left[1-\bar{w}_{\bar{y}_{i}}(\theta)\right]\right)\right]\left\{\begin{array}{c}
{\left[s+\mu_{2}\left[1-\bar{w}_{\bar{y}_{i}}(\theta)\right]\right]\left[\bar{w}_{U}{ }^{\prime}\left(s+\mu_{2}\left[1-\bar{w}_{\bar{y}_{i}}(\theta)\right]\right)\right]+} \\
{\left[1-\left[\bar{w}_{U}\left(s+\mu_{2}\left[1-\bar{w}_{\bar{y}_{i}}(\theta)\right]\right)\left\{\bar{w}_{\bar{x}_{i}}(\theta)\right\}\right]\right.}
\end{array}\right\}}
\end{array}\right\}}{\left\{\left[s+\mu_{2}\left[1-\bar{w}_{\bar{y}_{i}}(\theta)\right]\right]\left[1-\left[\bar{w}_{U}\left(s+\mu_{2}\left[1-\bar{w}_{\bar{y}_{i}}(\theta)\right]\right)\left\{\bar{w}_{\bar{x}_{i}}(\theta)\right\}\right]\right]\right\}^{2}}\right\} \\
\left\{\begin{array}{c}
\left\{\left[\mu_{2}\left[1-\bar{w}_{\bar{y}_{i}}(\theta)\right]\right]\left[\bar{w}_{U}{ }^{\prime}\left(\mu_{2}\left[1-\bar{w}_{\bar{y}_{i}}(\theta)\right]\right)\right]\right\}- \\
{\left[\frac{d}{d s}(A(s))\right]_{s=0}\left(\bar{w}_{U}\left(\mu_{2}\left[1-\bar{w}_{\bar{y}_{i}}(\theta)\right]\right)\right]\left\{\left[1-\left[\bar{w}_{U}\left(\mu_{2}\left[1-\bar{w}_{\bar{y}_{i}}(\theta)\right]\right)\left\{\bar{w}_{\bar{x}_{i}}(\theta)\right\}\right]\right]\right\}}
\end{array}\right\} \\
\left\{\left[\mu_{2}\left[1-\bar{w}_{\bar{y}_{i}}(\theta)\right]\right]\left[1-\left[\bar{w}_{U}\left(\mu_{2}\left[1-\bar{w}_{\bar{y}_{i}}(\theta)\right]\right)\left\{\bar{w}_{\bar{x}_{i}}(\theta)\right\}\right]\right]\right\}^{2}
\end{gathered}
$$

Again from equation (20) 
K.Srividhya and S.Sendhamizh Selvi

$$
\begin{gathered}
\frac{d}{d s}[B(s)]=\frac{\left\{\left[\bar{w}_{U}{ }^{\prime}\left(s+\mu_{2}\left[1-\bar{w}_{\bar{y}_{i}}(\theta)\right]\right)\right]\right\}}{\left\{1-\left[\bar{w}_{U}\left(s+\mu_{2}\left[1-\bar{w}_{\bar{y}_{i}}(\theta)\right]\right)\left\{\bar{w}_{\bar{x}_{i}}(\theta)\right\}\right]\right\}^{2}} \\
{\left[\frac{d}{d s}[B(s)]\right]_{s=0}=\frac{\left\{\left[\bar{w}_{U}\left(\mu_{2}\left[1-\bar{w}_{\bar{y}_{i}}(\theta)\right]\right)\right]\right\}}{\left\{1-\left[\bar{w}_{U}\left(\mu_{2}\left[1-\bar{w}_{\bar{y}_{i}}(\theta)\right]\right)\left\{\bar{w}_{\bar{x}_{i}}(\theta)\right\}\right]\right\}^{2}}}
\end{gathered}
$$

Using equations (21) and (22) in (19)

$$
\begin{aligned}
& {\left[\frac{d}{d s}\left[\bar{g}_{\theta}(s)\right]\right]_{s=0}=\frac{\left\{-1+\left[\bar{w}_{U}\left(\mu_{2}\left[1-\bar{w}_{\bar{y}_{i}}(\theta)\right]\right)\right]\right\}}{\left\{\mu_{2}\left[1-\bar{w}_{\bar{y}_{i}}(\theta)\right]\right\}\left\{\left[1-\left[\bar{w}_{U}\left(\mu_{2}\left[1-\bar{w}_{\bar{y}_{i}}(\theta)\right]\right)\left\{\bar{w}_{\bar{x}_{i}}(\theta)\right\}\right]\right]\right\}}} \\
& -\left[\frac{d}{d s}\left[\bar{g}_{\theta}(s)\right]\right]_{s=0}=\frac{\left\{1-\left[\bar{w}_{U}\left(\mu_{2}\left[1-\bar{w}_{\bar{y}_{i}}(\theta)\right]\right)\right]\right\}}{\left\{\mu_{2}\left[1-\bar{w}_{\bar{y}_{i}}(\theta)\right]\right\}\left\{\left[1-\left[\bar{w}_{U}\left(\mu_{2}\left[1-\bar{w}_{\bar{y}_{i}}(\theta)\right]\right)\left\{\bar{w}_{\bar{x}_{i}}(\theta)\right\}\right]\right]\right\}}(23) \\
& w_{U}(t)=p \mu_{h} e^{-\mu_{h} t}+(1-p) \mu_{l} e^{-\mu_{l} t} \\
& \quad \bar{w}_{U}(s)=\frac{p \mu_{h}}{s+\mu_{h}}+\frac{(1-p) \mu_{l}}{s+\mu_{l}} \\
& \left.\bar{w}_{U}{ }^{\prime}(s)=\frac{p \mu_{h}}{\left(s+\mu_{h}\right)^{2}}+\frac{(1-p) \mu_{l}}{\left(s+\mu_{l}\right)^{2}}\right\} \\
& \left.\left.\left.-\left[\frac{d}{d s}\left[\bar{g}_{\theta}(s)\right]\right]_{s=0}=\frac{p \mu_{h}}{\left\{\mu_{2}\left[1-\bar{w}_{\bar{y}_{i}}(\theta)\right]\right\}\left\{\left[1-\left[\frac{p \mu_{h}}{\mu_{2}\left[1-\bar{w}_{\bar{y}_{i}}(\theta)\right]+\mu_{h}}-\frac{(1-p) \mu_{l}}{\mu_{2}\left[1-\bar{w}_{\bar{y}_{i}}(\theta)\right]+\mu_{l}}\right.\right.\right.}+\frac{(1-p) \mu_{l}}{\left.\left.\mu_{\bar{y}_{i}}(\theta)\right]+\mu_{h}\left[1-\bar{w}_{\bar{y}_{i}}(\theta)\right]+\mu_{l}\right]}\right]\left\{\bar{w}_{\bar{x}_{i}}(\theta)\right\}\right]\right\}
\end{aligned}
$$

We know that $E(T)=-\left[\frac{d}{d s}\left[\bar{w}_{T}(s)\right]\right]_{s=0}$

From (12)

$$
\begin{gathered}
E(T)=-\sum_{i=r}^{n} n C_{i}\left\{p^{i}\left[\frac{d}{d s}\left[\bar{g}_{i\left(\theta+\theta_{1}\right)}(s)\right]\right]_{s=0}+\cdots+q^{i}\left[\frac{d}{d s}\left[\bar{g}_{i\left(\theta_{2}\right)}(s)\right]\right]_{s=0}\right\} \\
-(n-i) C_{1}\left\{p^{i}\left[\frac{d}{d s}\left[\bar{g}_{(i+1)\left(\theta+\theta_{1}\right)}(s)\right]\right]_{s=0}+\cdots+q^{i}\left[\frac{d}{d s}\left[\bar{g}_{(i+1)\left(\theta_{2}\right)}(s)\right]\right]_{s=0}\right\} \\
(-1)^{n}\left\{p^{n}\left[\frac{d}{d s}\left[\bar{g}_{n\left(\theta+\theta_{1}\right)}(s)\right]\right]_{s=0}+\cdots+q^{n}\left[\frac{d}{d s}\left[\bar{g}_{n\left(\theta_{2}\right)}(s)\right]\right]_{s=0}\right\}
\end{gathered}
$$

Since $\mathrm{x}_{\mathrm{i}}$ form an order statistics,

Let the probability function $x(1)$ and $x(m 1)$ are given by (Sheldon Ross 2005)

$\mathrm{w}_{\mathrm{x}(\mathrm{j})}(\mathrm{x})=\mathrm{j}\left(\begin{array}{c}\mathrm{m} 1 \\ \mathrm{j}\end{array}\right)\left[w_{x}(\mathrm{x})\right]^{\mathrm{j}-\mathrm{i}} w_{x}(\mathrm{x})\left[1-w_{x}(\mathrm{x})\right]^{\mathrm{m} 1-\mathrm{j}}, \mathrm{j}=1,2,3 \ldots \mathrm{m} 1$

Therefore the probability density function of $x(1)$ and $x(m 1)$ are given by

$\mathrm{w}_{\mathrm{x}(1)}(\mathrm{x})=m 1 w_{x}(x)\left[1-w_{x}(\mathrm{x})\right]^{\mathrm{m} 1-1}$

$\mathrm{w}_{\mathrm{X}_{(\mathrm{m} 1)}}(\mathrm{x})=m 1 w_{x}(x)\left[w_{x}(\mathrm{x})\right]^{\mathrm{m} 1-1}$

Similarly $\mathrm{y}_{\mathrm{j}}$ form an order statistics

Let the probability function $y(1)$ and $y(n 1)$ are given by (sheldonRoss 2005) 
Mean Time to Recruitment for a Multigrade Manpower System with Two Sources ...

$\mathrm{w}_{\mathrm{y}(\mathrm{j})}(\mathrm{y})=\mathrm{j}\left(\begin{array}{c}\mathrm{n} 1 \\ \mathrm{j}\end{array}\right)\left[w_{y}(\mathrm{y})\right]^{\mathrm{j}-\mathrm{i}} w_{y}(\mathrm{y})\left[1-w_{y}(\mathrm{y})\right]^{\mathrm{n} 1-\mathrm{j}}, \mathrm{j}=1,2,3 \ldots \mathrm{n} 1$

Therefore the probability density function of $y(1)$ and $y(n 1)$ are given by $\mathrm{w}_{\mathrm{y}(1)}(\mathrm{y})=$

$n 1 w_{y}(y)\left[1-w_{y}(\mathrm{y})\right]^{\mathrm{n} 1-1}$

$\mathrm{w}_{\mathrm{y}_{(\mathrm{n} 1)}}(\mathrm{y})=n 1 w_{y}(y)\left[w_{y}(\mathrm{y})\right]^{\mathrm{n} 1-1}$

\section{We shall now obtain the mean time to recruitment according as}

\section{Case (i)}

Suppose $w_{\bar{x}_{i}}(x)=\mathrm{w}_{\mathrm{x}(1)}(\mathrm{x})$ and $w_{\bar{y}_{i}}(y)=\mathrm{w}_{\mathrm{y}(1)}(\mathrm{y})$

Since $w_{x}(x)=c e^{-c x}$

$$
\begin{gathered}
w_{x(1)}(x)=m 1 c e^{-c x}\left(e^{-c x}\right)^{m 1-1}=m 1 c e^{-m 1 c x} \\
\bar{w}_{x(1)}(\theta)=m 1 c \int_{0}^{\infty} \mathrm{e}^{-\mathrm{m} 1 \mathrm{cx}} \mathrm{e}^{-\theta \mathrm{x}} \mathrm{dx}=\mathrm{m} 1 \mathrm{c} \int_{0}^{\infty} \mathrm{e}^{-(\mathrm{m} 1 \mathrm{c}+\theta) \mathrm{x}} \mathrm{dx}=\mathrm{m} 1 \mathrm{c}\left[\frac{\mathrm{e}^{-(\mathrm{m} 1 \mathrm{c}+\theta) \mathrm{x}}}{-(\mathrm{m} 1 \mathrm{c}+\theta)}\right]_{0}^{\infty} \\
=\frac{\mathrm{m} 1 \mathrm{c}}{\mathrm{m} 1 \mathrm{c}+\theta} \text { (33) }
\end{gathered}
$$

Similarly $\bar{w}_{y(1)}(\theta)=\frac{\mathrm{n} 1 \mathrm{c}}{\mathrm{n} 1 \mathrm{c}+\theta}$

Substituting (33) and (34) in (25)

$$
-\left[\frac{d}{d s}\left[\bar{g}_{\theta}(s)\right]\right]_{s=0}=\frac{1-\frac{p \mu_{h}}{\mu_{2}\left[1-\frac{\mathrm{n} 1 \mathrm{c}}{\mathrm{n} 1 \mathrm{c}+\theta}\right]+\mu_{h}}-\frac{(1-p) \mu_{l}}{\mu_{2}\left[1-\frac{\mathrm{n} 1 \mathrm{c}}{\mathrm{n} 1 \mathrm{c}+\theta}\right]+\mu_{l}}}{\left\{\mu_{2}\left[1-\frac{\mathrm{n} 1 \mathrm{c}}{\mathrm{n} 1 \mathrm{c}+\theta}\right]\right\}\left\{\left[1-\left[\frac{p \mu_{h}}{\mu_{2}\left[1-\frac{\mathrm{n} 1 \mathrm{c}}{\mathrm{n} 1 \mathrm{c}+\theta}\right]+\mu_{h}}+\frac{(1-p) \mu_{l}}{\mu_{2}\left[1-\frac{\mathrm{n} 1 \mathrm{c}}{\mathrm{n} 1 \mathrm{c}+\theta}\right]+\mu_{l}}\right]\left\{\frac{\mathrm{m} 1 \mathrm{c}}{\mathrm{m} 1 \mathrm{c}+\theta}\right\}\right]\right\}}
$$

Using (35) in (26), We get the mean time to recruitment.

\section{Case (ii)}

Suppose $w_{\bar{x}_{i}}(x)=w_{\mathbf{x}(1)}(x)$ and $w_{\bar{y}_{i}}(y)=w_{\mathbf{y}(n 1)}(y)$

Since $w_{y}(y)=c e^{-c y}$

Using equation (32)

$\mathbf{w}_{\mathbf{y}(\mathbf{n} 1)}(\mathbf{y})=n 1 c e^{-c y}\left(1-e^{-c y}\right)^{n 1-1}$

$\overline{\mathbf{w}}_{\mathbf{y}(\mathbf{n} 1)}(\boldsymbol{\theta})=\mathrm{n} 1 \mathrm{c} \int_{0}^{\infty} \mathrm{e}^{-\mathrm{cy}}\left(1-e^{-c y}\right)^{n 1-1} \mathrm{e}^{-\theta \mathrm{y}} \mathrm{dy}=\mathrm{n} 1 \int_{0}^{1}(1-\mathrm{z})^{\mathrm{n} 1-1} \mathrm{z}^{\frac{\theta}{c}} \mathrm{dz}$.

$=n 1 \beta\left(\frac{\theta}{c}+1, n 1\right)=n 1 \frac{\Gamma\left(\frac{\theta}{c}+1\right) \Gamma(n 1)}{\Gamma(\theta / c+n 1)}$

Simplifying the right side, we get $\quad \overline{\mathbf{w}}_{\mathbf{y}(\mathbf{n} \mathbf{1})}(\boldsymbol{\theta})=\frac{n 1 ! c^{n 1}}{\delta(c, \theta)}$

where $\delta(c, \theta)=(c+\theta)(2 c+\theta)(3 c+\theta) \ldots(n 1 c+\theta)$

Substituting (33) and (36) in (25) 
K.Srividhya and S.Sendhamizh Selvi

$$
-\left[\frac{d}{d s}\left[\bar{g}_{\theta}(s)\right]\right]_{s=0}=\frac{1-\frac{p \mu_{h}}{\mu_{2}\left[1-\frac{n 1 ! c^{n 1}}{\delta(c, \theta)}\right]+\mu_{h}}-\frac{(1-p) \mu_{l}}{\mu_{2}\left[1-\frac{n 1 ! c^{n 1}}{\delta(c, \theta)}\right]+\mu_{l}}}{\left\{\mu_{2}\left[1-\frac{n 1 ! c^{n 1}}{\delta(c, \theta)}\right]\right\}\left\{\left[1-\left[\frac{p \mu_{h}}{\mu_{2}\left[1-\frac{n 1 ! c^{n 1}}{\delta(c, \theta)}\right]+\mu_{h}}+\frac{(1-p) \mu_{l}}{\mu_{2}\left[1-\frac{n 1 ! c^{n 1}}{\delta(c, \theta)}\right]+\mu_{l}}\right]\left\{\frac{\mathrm{m} 1 \mathrm{c}}{\mathrm{m} 1 \mathrm{c}+\theta}\right\}\right]\right\}}
$$

where $\delta(c, \theta)$ is given by equation (37)

Using (38) in (26), We get the mean time to recruitment.

\section{Case (iii)}

Suppose $w_{\bar{x}_{i}}(x)=w_{x(m 1)}(x)$ and $w_{\bar{y}_{i}}(y)=w_{y(1)}(y)$

Using (36) for $\mathrm{x}$ we get $\overline{\mathbf{w}}_{\mathbf{X}(\mathbf{m} \mathbf{1})}(\boldsymbol{\theta})=\frac{m 1 ! c^{m 1}}{\delta(c, \theta)}$

where $\delta(c, \theta)$ is given by (37)

Substituting (34) and (39) in (25)

$$
-\left[\frac{d}{d s}\left[\bar{g}_{\theta}(s)\right]\right]_{s=0}=\frac{1-\frac{p \mu_{h}}{\mu_{2}\left[1-\frac{\mathrm{n} 1 \mathrm{c}}{\mathrm{n} 1 \mathrm{c}+\theta}\right]+\mu_{h}}-\frac{(1-p) \mu_{l}}{\mu_{2}\left[1-\frac{\mathrm{n} 1 \mathrm{c}}{\mathrm{n} 1 \mathrm{c}+\theta}\right]+\mu_{l}}}{\left\{\mu_{2}\left[1-\frac{\mathrm{n} 1 \mathrm{c}}{\mathrm{n} 1 \mathrm{c}+\theta}\right]\right\}\left\{\left[1-\left[\frac{p \mu_{h}}{\mu_{2}\left[1-\frac{\mathrm{n} 1 \mathrm{c}}{\mathrm{n} 1 \mathrm{c}+\theta}\right]+\mu_{h}}+\frac{(1-p) \mu_{l}}{\mu_{2}\left[1-\frac{\mathrm{n} 1 \mathrm{c}}{\mathrm{n} 1 \mathrm{c}+\theta}\right]+\mu_{l}}\right]\left\{\frac{m 1 ! c^{m 1}}{\delta(c, \theta)}\right\}\right]\right\}}
$$

where $\delta(c, \theta)$ is given by equation (37)

Using (40) in (26), We get the mean time to recruitment.

Case (iv)

Suppose $w_{\bar{x}_{i}}(x)=w_{x(m 1)}(x)$ and $w_{\bar{y}_{i}}(y)=w_{y(n 1)}(y)$

Substituting (39) and (36) in (25)

$$
-\left[\frac{d}{d s}\left[\bar{g}_{\theta}(s)\right]\right]_{s=0}=\frac{1-\frac{p \mu_{h}}{\mu_{2}\left[1-\frac{n 1 ! c^{n 1}}{\delta(c, \theta)}\right]+\mu_{h}}-\frac{(1-p) \mu_{l}}{\mu_{2}\left[1-\frac{n ! ! c^{n 1}}{\delta(c, \theta)}\right]+\mu_{l}}}{\left\{\mu_{2}\left[1-\frac{n 1 ! c^{n 1}}{\delta(c, \theta)}\right]\right\}\left\{1-\left[\frac{p \mu_{h}}{\mu_{2}\left[1-\frac{n ! c^{n 1}}{\delta(c, \theta)}\right]+\mu_{h}}+\frac{(1-p) \mu_{l}}{\mu_{2}\left[1-\frac{n 1 ! c}{\delta(c, \theta)}\right]+\mu_{l}}\right]\left\{\frac{m 1 ! c^{m 1}}{\delta(c, \theta)}\right\}\right\}}
$$

where $\delta(c, \theta)$ is given by equation (37)

Using (41) in (26), We get the mean time to recruitment.

\section{Numerical illustration}

The behavior of the performance measure due to the change in parameter is analyzed numerically for different values of $\mathrm{n}$ and $\mathrm{r}$.

Case (i)

Sub Case (i) $n=3, r=1$

The mean time to recruitment is given by,

$$
\begin{gathered}
E(T)=\left\{\left\{\left\{p^{3} E_{11}+3 p^{2} q^{1} E_{12}+3 p^{1} q^{2} E_{13}+q^{3} E_{14}\right\}\right\}-3\left\{\left\{p^{2} E_{15}+2 p q E_{16}+q^{2} E_{17}\right\}\right\}\right. \\
\left.+3\left\{\left\{p^{1} E_{18}+q^{1} E_{19}\right\}\right\}\right\}
\end{gathered}
$$


Mean Time to Recruitment for a Multigrade Manpower System with Two Sources ...

Sub Case (ii) $n=3, r=2$

The mean time to recruitment is given by

$E(T)=\left\{\left\{-2\left\{p^{3} E_{11}+3 p^{2} q^{1} E_{12}+3 p^{1} q^{2} E_{13}+q^{3} E_{14}\right\}\right\}+3\left\{\left\{p^{2} E_{15}+2 p q E_{16}+q^{2} E_{17}\right\}\right\}\right\}$

Sub Case (iii) $n=3, r=3$

The mean time to recruitment is given by

where

$$
E(T)=\left\{\left\{p^{3} E_{11}+3 p^{2} q^{1} E_{12}+3 p^{1} q^{2} E_{13}+q^{3} E_{14}\right\}\right\}
$$

$E_{11}=\frac{1-\frac{p \mu_{h}}{\mu_{2}\left[1-\frac{\mathrm{n} 1 \mathrm{c}}{\mathrm{n} 1 \mathrm{c}+3\left(\theta+\theta_{1}\right)}\right]+\mu_{h}}-\frac{(1-p) \mu_{l}}{\mu_{2}\left[1-\frac{\mathrm{ncc}}{\mathrm{n} 1 \mathrm{c}+3\left(\theta+\theta_{1}\right)}\right]+\mu_{l}}}{\left\{\mu_{2}\left[1-\frac{\mathrm{n} 1 \mathrm{c}}{\mathrm{n} 1 \mathrm{c}+3\left(\theta+\theta_{1}\right)}\right]\right\}\left\{\left[1-\left[\frac{p \mu_{h}}{\mu_{2}\left[1-\frac{\mathrm{n} 1 \mathrm{c}}{\mathrm{n} 1 \mathrm{c}+3\left(\theta+\theta_{1}\right)}\right]+\mu_{h}}+\frac{(1-p) \mu_{l}}{\mu_{2}\left[1-\frac{\mathrm{n} 1 \mathrm{c}}{\mathrm{n} 1 \mathrm{c}+3\left(\theta+\theta_{1}\right)}\right]+\mu_{l}}\right]\left\{\frac{\mathrm{m} 1 \mathrm{c}}{\mathrm{m} 1 \mathrm{c}+3\left(\theta+\theta_{1}\right)}\right]\right\}\right.}$

$E_{12}$

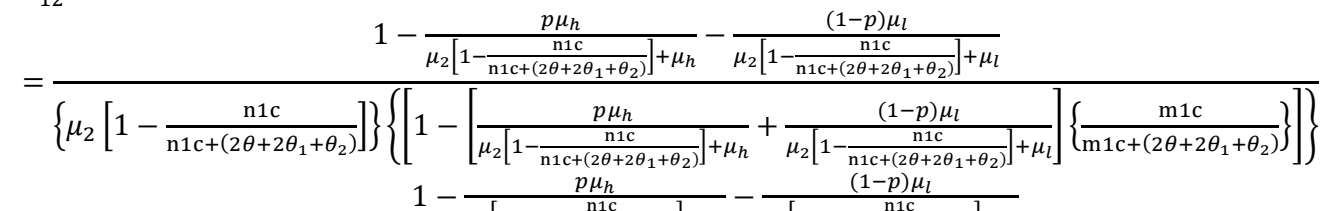

$E_{13}=\frac{1-\frac{p \mu_{h}}{\mu_{2}\left[1-\frac{\mathrm{n} 1 \mathrm{c}}{\mathrm{n} 1 \mathrm{c}+\left(\theta+\theta_{1}+2 \theta_{2}\right)}\right]+\mu_{h}}-\frac{(1-p) \mu_{l}}{\mu_{2}\left[1-\frac{\mathrm{n} 1 \mathrm{c}}{\mathrm{n} 1 \mathrm{c}+\left(\theta+\theta_{1}+2 \theta_{2}\right)}\right]+\mu_{l}}}{\left.\left\{\mu_{2}\left[1-\frac{\mathrm{n} 1 \mathrm{c}}{\mathrm{n} 1 \mathrm{c}+\left(\theta+\theta_{1}+2 \theta_{2}\right)}\right]\right\}\left\{1-\left[\frac{p \mu_{h}}{\mu_{2}\left[1-\frac{\mathrm{n} 1 \mathrm{c}}{\mathrm{n} 1 \mathrm{c}+\left(\theta+\theta_{1}+2 \theta_{2}\right)}\right]+\mu_{h}}+\frac{(1-p) \mu_{l}}{\mu_{2}\left[1-\frac{\mathrm{n} 1 \mathrm{c}}{\mathrm{n} 1 \mathrm{c}+\left(\theta+\theta_{1}+2 \theta_{2}\right)}\right]+\mu_{l}}\right]\left\{\frac{\mathrm{m} 1 \mathrm{c}}{\mathrm{m} 1 \mathrm{c}+\left(\theta+\theta_{1}+2 \theta_{2}\right)}\right\}\right]\right\}}$

$1-\frac{p \mu_{h}}{\mu_{2}\left[1-\frac{\mathrm{n} 1 \mathrm{c}}{\mathrm{n} 1 \mathrm{c}+3\left(\theta_{2}\right)}\right]+\mu_{h}}-\frac{(1-p) \mu_{l}}{\mu_{2}\left[1-\frac{\mathrm{n} 1 \mathrm{c}}{\mathrm{n} 1 \mathrm{c}+3\left(\theta_{2}\right)}\right]+\mu_{l}}$

$E_{14}=\frac{\mu_{2}\left[1-\frac{\mathrm{n} 1 \mathrm{c}}{\mathrm{n} 1 \mathrm{c}+3\left(\theta_{2}\right)}\right]+\mu_{h} \quad \mu_{2}\left[1-\frac{\mathrm{n} 1 \mathrm{c}}{\mathrm{n} 1 \mathrm{c}+3\left(\theta_{2}\right)}\right]+\mu_{l}}{\left\{\mu_{2}\left[1-\frac{\mathrm{n} 1 \mathrm{c}}{\mathrm{n} 1 \mathrm{c}+3\left(\theta_{2}\right)}\right]\right\}\left\{\left[1-\left[\frac{p \mu_{h}}{\mu_{2}\left[1-\frac{\mathrm{n} 1 \mathrm{c}}{\mathrm{n} 1 \mathrm{c}+3\left(\theta_{2}\right)}\right]+\mu_{h}}+\frac{(1-p) \mu_{l}}{\mu_{2}\left[1-\frac{\mathrm{n} 1 \mathrm{c}}{\mathrm{n} 1 \mathrm{c}+3\left(\theta_{2}\right)}\right]+\mu_{l}}\right]\left\{\frac{\mathrm{m} 1 \mathrm{c}}{\mathrm{m} 1 \mathrm{c}+3\left(\theta_{2}\right)}\right\}\right]\right\}}$

$1-\frac{p \mu_{h}}{\mu_{2}\left[1-\frac{\mathrm{n} 1 \mathrm{c}}{\mathrm{n} 1 \mathrm{c}+2\left(\theta+\theta_{1}\right)}\right]+\mu_{h}}-\frac{(1-p) \mu_{l}}{\mu_{2}\left[1-\frac{\mathrm{n} 1 \mathrm{c}}{\mathrm{n} 1 \mathrm{c}+2\left(\theta+\theta_{1}\right)}\right]+\mu_{l}}$

$E_{15}=\frac{\left\{\mu_{2}\left[1-\frac{\mathrm{n} 1 \mathrm{c}}{\mathrm{n} 1 \mathrm{c}+2\left(\theta+\theta_{1}\right)}\right]\right\}\left\{\left[1-\left[\frac{p \mu_{h}}{\mu_{2}\left[1-\frac{\mathrm{n} 1 \mathrm{c}}{\mathrm{n} 1 \mathrm{c}+2\left(\theta+\theta_{1}\right)}\right]+\mu_{h}}+\frac{(1-p) \mu_{l}}{\mu_{2}\left[1-\frac{\mathrm{n} 1 \mathrm{c}}{\mathrm{n} 1 \mathrm{c}+2\left(\theta+\theta_{1}\right)}\right]+\mu_{l}}\right]\left\{\frac{\mathrm{m} 1 \mathrm{c}}{\mathrm{m} 1 \mathrm{c}+2\left(\theta+\theta_{1}\right)}\right\}\right]\right\}}{\{}$

$E_{16}=\frac{1-\frac{p \mu_{h}}{\mu_{2}\left[1-\frac{\mathrm{n} 1 \mathrm{c}}{\mathrm{n} 1 \mathrm{c}+\left(\theta+\theta_{1}+\theta_{2}\right)}\right]+\mu_{h}}-\frac{(1-p) \mu_{l}}{\mu_{2}\left[1-\frac{\mathrm{n} 1 \mathrm{c}}{\mathrm{n} 1 \mathrm{c}+\left(\theta+\theta_{1}+\theta_{2}\right)}\right]+\mu_{l}}}{\left.\left\{\mu_{2}\left[1-\frac{\mathrm{n} 1 \mathrm{c}}{\mathrm{n} 1 \mathrm{c}+\left(\theta+\theta_{1}+\theta_{2}\right)}\right]\right\}\left\{1-\left[\frac{p \mu_{h}}{\mu_{2}\left[1-\frac{\mathrm{n} 1 \mathrm{c}}{\mathrm{n} 1 \mathrm{c}+\left(\theta+\theta_{1}+\theta_{2}\right)}\right]+\mu_{h}}+\frac{(1-p) \mu_{l}}{\mu_{2}\left[1-\frac{\mathrm{n} 1 \mathrm{c}}{\mathrm{n} 1 \mathrm{c}+\left(\theta+\theta_{1}+\theta_{2}\right)}\right]+\mu_{l}}\right]\left\{\frac{\mathrm{m} 1 \mathrm{c}}{\mathrm{m} 1 \mathrm{c}+\left(\theta+\theta_{1}+\theta_{2}\right)}\right\}\right]\right\}}$

$1-\frac{p \mu_{h}}{\mu_{2}\left[1-\frac{\mathrm{n} 1 \mathrm{c}}{\mathrm{n} 1 \mathrm{c}+\left(2 \theta_{2}\right)}\right]+\mu_{h}}-\frac{(1-p) \mu_{l}}{\mu_{2}\left[1-\frac{\mathrm{n} 1 \mathrm{c}}{\mathrm{n} 1 \mathrm{c}+\left(2 \theta_{2}\right)}\right]+\mu_{l}}$

$E_{17}=\frac{\left\{\mu_{2}\left[1-\frac{\mathrm{n} 1 \mathrm{c}}{\mathrm{n} 1 \mathrm{c}+\left(2 \theta_{2}\right)}\right]\right\}\left\{\left[1-\left[\frac{p \mu_{h}}{\mu_{2}\left[1-\frac{\mathrm{n} 1 \mathrm{c}}{\mathrm{n} 1 \mathrm{c}+\left(2 \theta_{2}\right)}\right]+\mu_{h}}+\frac{(1-p) \mu_{l}}{\mu_{2}\left[1-\frac{\mathrm{n} 1 \mathrm{c}}{\mathrm{n} 1 \mathrm{c}+\left(2 \theta_{2}\right)}\right]+\mu_{l}}\right]\left\{\frac{\mathrm{m} 1 \mathrm{c}}{\mathrm{m} 1 \mathrm{c}+\left(2 \theta_{2}\right)}\right\}\right]\right\}}{(-p) \mu_{l}}$

$E_{18}=\frac{1-\frac{p \mu_{h}}{\mu_{2}\left[1-\frac{\mathrm{n} 1 \mathrm{c}}{\mathrm{n} 1 \mathrm{c}+\left(\theta+\theta_{1}\right)}\right]+\mu_{h}}-\frac{(1-p) \mu_{l}}{\mu_{2}\left[1-\frac{\mathrm{n} 1 \mathrm{c}}{\mathrm{n} 1 \mathrm{c}+\left(\theta+\theta_{1}\right)}\right]+\mu_{l}}}{\left.\left\{\mu_{2}\left[1-\frac{\mathrm{n} 1 \mathrm{c}}{\mathrm{n} 1 \mathrm{c}+\left(\theta+\theta_{1}\right)}\right]\right\}\left\{1-\left[\frac{p \mu_{h}}{\mu_{2}\left[1-\frac{\mathrm{n} 1 \mathrm{c}}{\mathrm{n} 1 \mathrm{c}+\left(\theta+\theta_{1}\right)}\right]+\mu_{h}}+\frac{(1-p) \mu_{l}}{\mu_{2}\left[1-\frac{\mathrm{n} 1 \mathrm{c}}{\mathrm{n} 1 \mathrm{c}+\left(\theta+\theta_{1}\right)}\right]+\mu_{l}}\right]\left\{\frac{\mathrm{m} 1 \mathrm{c}}{\mathrm{m} 1 \mathrm{c}+\left(\theta+\theta_{1}\right)}\right\}\right]\right\}}$ 
K.Srividhya and S.Sendhamizh Selvi

$E_{19}=\frac{1-\frac{p \mu_{h}}{\mu_{2}\left[1-\frac{\mathrm{n} 1 \mathrm{c}}{\mathrm{n} 1 \mathrm{c}+\left(\theta_{2}\right)}\right]+\mu_{h}}-\frac{(1-p) \mu_{l}}{\mu_{2}\left[1-\frac{\mathrm{n} 1 \mathrm{c}}{\mathrm{n} 1 \mathrm{c}+\left(\theta_{2}\right)}\right]+\mu_{l}}}{\left\{\mu_{2}\left[1-\frac{\mathrm{n} 1 \mathrm{c}}{\mathrm{n} 1 \mathrm{c}+\left(\theta_{2}\right)}\right]\right\}\left\{\left[1-\left[\frac{p \mu_{h}}{\mu_{2}\left[1-\frac{\mathrm{n} 1 \mathrm{c}}{\mathrm{n} 1 \mathrm{c}+\left(\theta_{2}\right)}\right]+\mu_{h}}+\frac{(1-p) \mu_{l}}{\mu_{2}\left[1-\frac{\mathrm{n} 1 \mathrm{c}}{\mathrm{n} 1 \mathrm{c}+\left(\theta_{2}\right)}\right]+\mu_{l}}\right]\left\{\frac{\mathrm{m} 1 \mathrm{c}}{\mathrm{m} 1 \mathrm{c}+\left(\theta_{2}\right)}\right\}\right]\right\}}$

Case (ii)

Sub Case (i) $n=3, r=1$

The mean time to recruitment is given by,

$$
\begin{aligned}
E(T)=\left\{\left\{\left\{p^{3} E_{21}\right.\right.\right. & \left.\left.+3 p^{2} q^{1} E_{22}+3 p^{1} q^{2} E_{23}+q^{3} E_{24}\right\}\right\}-3\left\{\left\{p^{2} E_{25}+2 p q E_{26}+q^{2} E_{27}\right\}\right\} \\
& \left.+3\left\{\left\{p^{1} E_{28}+q^{1} E_{29}\right\}\right\}\right\}
\end{aligned}
$$

Sub Case (ii) $n=3, r=2$

The mean time to recruitment is given by

$$
E(T)=\left\{\left\{-2\left\{p^{3} E_{21}+3 p^{2} q^{1} E_{22}+3 p^{1} q^{2} E_{23}+q^{3} E_{24}\right\}\right\}+3\left\{\left\{p^{2} E_{25}+2 p q E_{26}+q^{2} E_{27}\right\}\right\}\right\}
$$

Sub Case (iii) $n=3, r=3$

The mean time to recruitment is given by

where

$$
E(T)=\left\{\left\{\left\{p^{3} E_{21}+3 p^{2} q^{1} E_{22}+3 p^{1} q^{2} E_{23}+q^{3} E_{24}\right\}\right\}\right\}
$$

$$
\begin{aligned}
& E_{21}=\frac{1-\frac{p \mu_{h}}{\mu_{2}\left[1-\frac{n 1 ! c^{n 1}}{\delta\left(c, 3\left(\theta+\theta_{1}\right)\right)}\right]+\mu_{h}}-\frac{(1-p) \mu_{l}}{\mu_{2}\left[1-\frac{n 1 ! c^{n 1}}{\delta\left(c, 3\left(\theta+\theta_{1}\right)\right)}\right]+\mu_{l}}}{\left.\left\{\mu_{2}\left[1-\frac{n 1 ! c^{n 1}}{\delta\left(c, 3\left(\theta+\theta_{1}\right)\right)}\right]\right\}\left\{1-\left[\frac{p \mu_{h}}{\mu_{2}\left[1-\frac{n 1 ! c^{n 1}}{\delta\left(c, 3\left(\theta+\theta_{1}\right)\right.}\right]+\mu_{h}}+\frac{(1-p) \mu_{l}}{\mu_{2}\left[1-\frac{n 1 ! c^{n 1}}{\delta\left(c, 3\left(\theta+\theta_{1}\right)\right)}\right]+\mu_{l}}\right]\left\{\frac{\mathrm{m} 1 \mathrm{c}}{\mathrm{m} 1 \mathrm{c}+3\left(\theta+\theta_{1}\right)}\right\}\right]\right\}} \\
& 1-\frac{p \mu_{h}}{\mu_{2}\left[1-\frac{n 1 ! c^{n 1}}{\delta\left(c,\left(2 \theta+2 \theta_{1}+\theta_{2}\right)\right)}\right]+\mu_{h}}-\frac{(1-p) \mu_{l}}{\mu_{2}\left[1-\frac{n 1 ! c^{n 1}}{\delta\left(c,\left(2 \theta+2 \theta_{1}+\theta_{2}\right)\right.}\right]+\mu_{l}}
\end{aligned}
$$

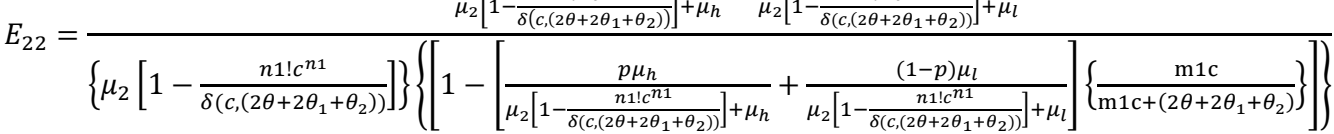

$$
\begin{aligned}
& 1-\frac{p \mu_{h}}{\mu_{2}\left[1-\frac{n 1 ! c^{n 1}}{\delta\left(c,\left(\theta+\theta_{1}+2 \theta_{2}\right)\right)}\right]+\mu_{h}}-\frac{(1-p) \mu_{l}}{\mu_{2}\left[1-\frac{n 1 ! c^{n 1}}{\delta\left(c,\left(\theta+\theta_{1}+2 \theta_{2}\right)\right)}\right]+\mu_{l}} \\
& E_{23}=\frac{\mu_{2}\left[1-\frac{n\left(c,\left(\theta+\theta_{1}+2 \theta_{2}\right)\right)}{\delta\left(\mu_{h}\right.}\right]}{\left\{\mu_{2}\left[1-\frac{n 1 ! c^{n 1}}{\delta\left(c,\left(\theta+\theta_{1}+2 \theta_{2}\right)\right)}\right]\right\}\left\{\left[1-\left[\frac{p \mu_{h}}{\mu_{2}\left[1-\frac{n 1 ! c^{n 1}}{\delta\left(c,\left(\theta+\theta_{1}+2 \theta_{2}\right)\right.}\right]+\mu_{h}}+\frac{(1-p) \mu_{l}}{\mu_{2}\left[1-\frac{n 1 ! c^{n 1}}{\delta\left(c,\left(\theta+\theta_{1}+2 \theta_{2}\right)\right.}\right]+\mu_{l}}\right]\left\{\frac{\mathrm{m} 1 \mathrm{c}}{\mathrm{m} 1 \mathrm{c}+\left(\theta+\theta_{1}+2 \theta_{2}\right)}\right\}\right]\right\}} \\
& 1-\frac{p \mu_{h}}{\mu_{2}\left[1-\frac{n 1 ! c^{n 1}}{\delta\left(c, 3\left(\theta_{2}\right)\right)}\right]+\mu_{h}}-\frac{(1-p) \mu_{l}}{\mu_{2}\left[1-\frac{n 1 ! c^{n 1}}{\delta\left(c, 3\left(\theta_{2}\right)\right)}\right]+\mu_{l}}
\end{aligned}
$$

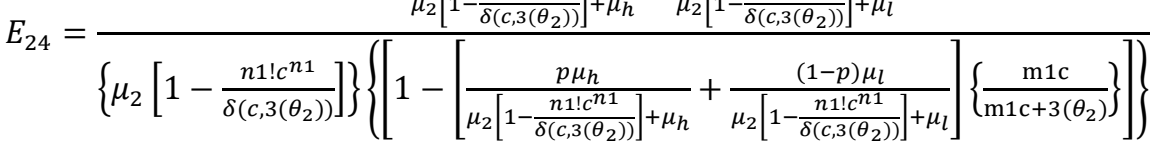

$$
\begin{aligned}
& 1-\frac{p \mu_{h}}{\mu_{2}\left[1-\frac{n 1 ! c^{n 1}}{\delta\left(c, 2\left(\theta+\theta_{1}\right)\right)}\right]+\mu_{h}}-\frac{(1-p) \mu_{l}}{\mu_{2}\left[1-\frac{n 1 ! c^{n 1}}{\delta\left(c, 2\left(\theta+\theta_{1}\right)\right)}\right]+\mu_{l}} \\
& E_{25}=\frac{\mu_{2}\left[1-\frac{n ! c^{n}}{\delta\left(c, 2\left(\theta+\theta_{1}\right)\right)}\right]+\mu_{h} \quad \mu_{2}\left[1-\frac{n 1 \cdot c}{\delta\left(c, 2\left(\theta+\theta_{1}\right)\right)}\right]+\mu_{l}}{\left\{\mu_{2}\left[1-\frac{n 1 ! c^{n 1}}{\delta\left(c, 2\left(\theta+\theta_{1}\right)\right)}\right]\right\}\left\{\left[-\left[\frac{p \mu_{h}}{\mu_{2}\left[1-\frac{n 1 ! c^{n 1}}{\delta\left(c, 2\left(\theta+\theta_{1}\right)\right)}\right]+\mu_{h}}+\frac{(1-p) \mu_{l}}{\mu_{2}\left[1-\frac{n 1 ! c^{n 1}}{\delta\left(c, 2\left(\theta+\theta_{1}\right)\right.}\right]+\mu_{l}}\right]\left\{\frac{\mathrm{m} 1 \mathrm{c}}{\mathrm{m} 1 \mathrm{c}+2\left(\theta+\theta_{1}\right)}\right\}\right]\right\}} \\
& 1-\frac{p \mu_{h}}{\mu_{2}\left[1-\frac{n 1 ! c^{n 1}}{\delta\left(c,\left(\theta+\theta_{1}+\theta_{2}\right)\right)}\right]+\mu_{h}}-\frac{(1-p) \mu_{l}}{\mu_{2}\left[1-\frac{n 1 ! c^{n 1}}{\delta\left(c,\left(\theta+\theta_{1}+\theta_{2}\right)\right)}\right]+\mu_{l}} \\
& \left.\left.E_{26}=\frac{\left.\mu_{2}\left[1-\frac{n 1 ! c^{n 1}}{\delta\left(c,\left(\theta+\theta_{1}+\theta_{2}\right)\right)}\right]\right\}\left\{\left[1-\left[\frac{p \mu_{h}}{\mu_{2}\left[1-\frac{n 1 ! c^{n 1}}{\delta\left(c,\left(\theta+\theta_{1}+\theta_{2}\right)\right.}\right]+\mu_{h}}+\frac{(1-p) \mu_{l}}{\mu_{2}\left[1-\frac{n 1 ! c^{n 1}}{\delta\left(c,\left(\theta+\theta_{1}+\theta_{2}\right)\right)}\right]+\mu_{l}}\right]\left\{\frac{\mathrm{m} 1 \mathrm{c}}{\mathrm{m} 1 \mathrm{c}+\left(\theta+\theta_{1}+\theta_{2}\right)}\right\}\right]\right\}}{\delta\left(c,\left(\theta+\theta_{1}+\theta_{2}\right)\right)}\right\}\right)
\end{aligned}
$$


Mean Time to Recruitment for a Multigrade Manpower System with Two Sources ...

$$
\begin{aligned}
& 1-\frac{p \mu_{h}}{\mu_{2}\left[1-\frac{n 1 ! c^{n 1}}{\delta\left(c, 2\left(\theta_{2}\right)\right)}\right]+\mu_{h}}-\frac{(1-p) \mu_{l}}{\mu_{2}\left[1-\frac{n 1 ! c^{n 1}}{\delta\left(c, 2\left(\theta_{2}\right)\right)}\right]+\mu_{l}} \\
& \left.\left.E_{27}=\frac{\left.\mu_{2}\left[1-\frac{n 1 ! c^{n 1}}{\delta\left(c, 2\left(\theta_{2}\right)\right)}\right]\right\}\left\{\left[1-\left[\frac{p \mu_{h}}{\mu_{2}\left[1-\frac{n 1 ! c^{n 1}}{\delta\left(c, 2\left(\theta_{2}\right)\right)}\right]+\mu_{h}}+\frac{(1-p) \mu_{l}}{\mu_{2}\left[1-\frac{n 1 ! c^{n 1}}{\delta\left(c, 2\left(\theta_{2}\right)\right)}\right]+\mu_{l}}\right]\left\{\frac{\mathrm{m} 1 \mathrm{c}}{\mathrm{m} 1 \mathrm{c}+2\left(\theta_{2}\right)}\right\}\right]\right\}}{\delta\left(c, 2\left(\theta_{2}\right)\right)}\right\}\right) \\
& 1-\frac{p \mu_{h}}{\mu_{2}\left[1-\frac{n 1 ! c^{n 1}}{\delta\left(c,\left(\theta+\theta_{1}\right)\right)}\right]+\mu_{h}}-\frac{(1-p) \mu_{l}}{\mu_{2}\left[1-\frac{n 1 ! c^{n 1}}{\delta\left(c,\left(\theta+\theta_{1}\right)\right.}\right]+\mu_{l}} \\
& E_{28}=\frac{\mu_{2}\left[1-\frac{\left(c,\left(\theta+\theta_{1}\right)\right)}{\delta\left(\mu_{h}\right.} \mu_{2}\left[1-\frac{p\left(c,\left(\theta+\theta_{1}\right)\right)}{\delta\left(\mu_{l}\right.}\right.\right.}{\left\{\mu_{2}\left[1-\frac{n 1 ! c^{n 1}}{\delta\left(c,\left(\theta+\theta_{1}\right)\right)}\right]\right\}\left\{\left[1-\left[\frac{p \mu_{h}}{\mu_{2}\left[1-\frac{n 1 ! c^{n 1}}{\delta\left(c,\left(\theta+\theta_{1}\right)\right)}\right]+\mu_{h}}+\frac{(1-p) \mu_{l}}{\mu_{2}\left[1-\frac{n 1 ! c^{n 1}}{\delta\left(c,\left(\theta+\theta_{1}\right)\right)}\right]+\mu_{l}}\right]\left\{\frac{\mathrm{m} 1 \mathrm{c}}{\mathrm{m} 1 \mathrm{c}+\left(\theta+\theta_{1}\right)}\right\}\right]\right\}} \\
& 1-\frac{p \mu_{h}}{\mu_{2}\left[1-\frac{n 1 ! c^{n 1}}{\delta\left(c,\left(\theta_{2}\right)\right)}\right]+\mu_{h}}-\frac{(1-p) \mu_{l}}{\mu_{2}\left[1-\frac{n 1 ! c^{n 1}}{\delta\left(c,\left(\theta_{2}\right)\right)}\right]+\mu_{l}}
\end{aligned}
$$

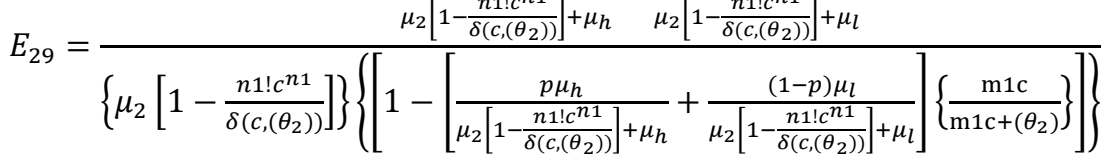

\section{Case (iii)}

Sub Case (i) $n=3, r=1$

The mean time to recruitment is given by,

$$
\begin{aligned}
E(T)=\left\{\left\{\left\{p^{3} E_{31}+3 p^{2} q^{1} E_{32}+3 p^{1} q^{2} E_{33}+q^{3} E_{34}\right\}\right\}-3\left\{\left\{p^{2} E_{35}+2 p q E_{36}+q^{2} E_{37}\right\}\right\}\right. \\
\left.+3\left\{\left\{p^{1} E_{38}+q^{1} E_{39}\right\}\right\}\right\}
\end{aligned}
$$

Sub Case (ii) $n=3, r=2$

The mean time to recruitment is given by

$$
E(T)=\left\{\left\{-2\left\{p^{3} E_{31}+3 p^{2} q^{1} E_{32}+3 p^{1} q^{2} E_{33}+q^{3} E_{34}\right\}\right\}+3\left\{\left\{p^{2} E_{35}+2 p q E_{36}+q^{2} E_{37}\right\}\right\}\right\}
$$

Sub Case (iii) $n=3, r=3$

The mean time to recruitment is given by

where

$$
E(T)=\left\{\left\{\left\{p^{3} E_{31}+3 p^{2} q^{1} E_{32}+3 p^{1} q^{2} E_{33}+q^{3} E_{34}\right\}\right\}\right\}
$$

$$
\begin{aligned}
& E_{31}=\frac{1-\frac{p \mu_{h}}{\mu_{2}\left[1-\frac{\mathrm{n} 1 \mathrm{c}}{\mathrm{n} 1 \mathrm{c}+3\left(\theta+\theta_{1}\right)}\right]+\mu_{h}}-\frac{(1-p) \mu_{l}}{\mu_{2}\left[1-\frac{\mathrm{n} 1 \mathrm{c}}{\mathrm{n} 1 \mathrm{c}+3\left(\theta+\theta_{1}\right)}\right]+\mu_{l}}}{\left\{\mu_{2}\left[1-\frac{\mathrm{n} 1 \mathrm{c}}{\mathrm{n} 1 \mathrm{c}+3\left(\theta+\theta_{1}\right)}\right]\right\}\left\{\left[1-\left[\frac{p \mu_{h}}{\mu_{2}\left[1-\frac{\mathrm{n} 1 \mathrm{c}}{\mathrm{n} 1 \mathrm{c}+3\left(\theta+\theta_{1}\right)}\right]+\mu_{h}}+\frac{(1-p) \mu_{l}}{\mu_{2}\left[1-\frac{\mathrm{n} 1 \mathrm{c}}{\mathrm{n} 1 \mathrm{c}+3\left(\theta+\theta_{1}\right)}\right]+\mu_{l}}\right]\left\{\frac{m 1 ! c m 1}{\delta\left(c, 3\left(\theta+\theta_{1}\right)\right)}\right\}\right]\right\}} \\
& E_{32}=\frac{1-\frac{p \mu_{h}}{\mu_{2}\left[1-\frac{\mathrm{n} 1 \mathrm{c}}{\mathrm{n} 1 \mathrm{c}+\left(2 \theta+2 \theta_{1}+\theta_{2}\right)}\right]+\mu_{h}}-\frac{(1-p) \mu_{l}}{\mu_{2}\left[1-\frac{\mathrm{n} 1 \mathrm{c}}{\mathrm{n} 1 \mathrm{c}+\left(2 \theta+2 \theta_{1}+\theta_{2}\right)}\right]+\mu_{l}}}{\left.\left\{\mu_{2}\left[1-\frac{\mathrm{n} 1 \mathrm{c}}{\mathrm{n} 1 \mathrm{c}+\left(2 \theta+2 \theta_{1}+\theta_{2}\right)}\right]\right\}\left\{1-\left[\frac{p \mu_{h}}{\mu_{2}\left[1-\frac{\mathrm{n} 1 \mathrm{c}}{\mathrm{n} 1 \mathrm{c}+\left(2 \theta+2 \theta_{1}+\theta_{2}\right)}\right]+\mu_{h}}+\frac{(1-p) \mu_{l}}{\mu_{2}\left[1-\frac{\mathrm{n} 1 \mathrm{c}}{\mathrm{n} 1 \mathrm{c}+\left(2 \theta+2 \theta_{1}+\theta_{2}\right)}\right]+\mu_{l}}\right]\left\{\frac{m 1 ! c^{m 1}}{\delta\left(c,\left(2 \theta+2 \theta_{1}+\theta_{2}\right)\right)}\right\}\right]\right\}} \\
& E_{33}=\frac{1-\frac{p \mu_{h}}{\mu_{2}\left[1-\frac{\mathrm{n} 1 \mathrm{c}}{\mathrm{n} 1 \mathrm{c}+\left(\theta+\theta_{1}+2 \theta_{2}\right)}\right]+\mu_{h}}-\frac{(1-p) \mu_{l}}{\mu_{2}\left[1-\frac{\mathrm{n} 1 \mathrm{c}}{\mathrm{n} 1 \mathrm{c}+\left(\theta+\theta_{1}+2 \theta_{2}\right)}\right]+\mu_{l}}}{\left\{\mu_{2}\left[1-\frac{\mathrm{n} 1 \mathrm{c}}{\mathrm{n} 1 \mathrm{c}+\left(\theta+\theta_{1}+2 \theta_{2}\right)}\right]\right\}\left\{\left[1-\left[\frac{p \mu_{h}}{\mu_{2}\left[1-\frac{\mathrm{n} 1 \mathrm{c}}{\mathrm{n} 1 \mathrm{c}+\left(\theta+\theta_{1}+2 \theta_{2}\right)}\right]+\mu_{h}}+\frac{(1-p) \mu_{l}}{\mu_{2}\left[1-\frac{\mathrm{n} 1 \mathrm{c}}{\mathrm{n} 1 \mathrm{c}+\left(\theta+\theta_{1}+2 \theta_{2}\right)}\right]+\mu_{l}}\right]\left\{\frac{m 1 ! c m 1}{\delta\left(c,\left(\theta+\theta_{1}+2 \theta_{2}\right)\right)}\right\}\right]\right\}} \\
& E_{34}=\frac{1-\frac{p \mu_{h}}{\mu_{2}\left[1-\frac{\mathrm{n} 1 \mathrm{c}}{\mathrm{n} 1 \mathrm{c}+3\left(\theta_{2}\right)}\right]+\mu_{h}}-\frac{(1-p) \mu_{l}}{\mu_{2}\left[1-\frac{\mathrm{n} 1 \mathrm{c}}{\mathrm{n} 1 \mathrm{c}+3\left(\theta_{2}\right)}\right]+\mu_{l}}}{\left\{\mu_{2}\left[1-\frac{\mathrm{n} 1 \mathrm{c}}{\mathrm{n} 1 \mathrm{c}+3\left(\theta_{2}\right)}\right]\right\}\left\{\left[1-\left[\frac{p \mu_{h}}{\mu_{2}\left[1-\frac{\mathrm{n} 1 \mathrm{c}}{\mathrm{n} 1 \mathrm{c}+3\left(\theta_{2}\right)}\right]+\mu_{h}}+\frac{(1-p) \mu_{l}}{\mu_{2}\left[1-\frac{\mathrm{n} 1 \mathrm{c}}{\mathrm{n} 1 \mathrm{c}+3\left(\theta_{2}\right)}\right]+\mu_{l}}\right]\left\{\frac{m 1 ! c m 1}{\delta\left(c, 3\left(\theta_{2}\right)\right)}\right\}\right]\right\}}
\end{aligned}
$$


K.Srividhya and S.Sendhamizh Selvi

$$
\begin{aligned}
& E_{35}=\frac{1-\frac{p \mu_{h}}{\mu_{2}\left[1-\frac{\mathrm{n} 1 \mathrm{c}}{\mathrm{n} 1 \mathrm{c}+2\left(\theta+\theta_{1}\right)}\right]+\mu_{h}}-\frac{(1-p) \mu_{l}}{\mu_{2}\left[1-\frac{\mathrm{n} 1 \mathrm{c}}{\mathrm{n} 1 \mathrm{c}+2\left(\theta+\theta_{1}\right)}\right]+\mu_{l}}}{\left\{\mu_{2}\left[1-\frac{\mathrm{n} 1 \mathrm{c}}{\mathrm{n} 1 \mathrm{c}+2\left(\theta+\theta_{1}\right)}\right]\right\}\left\{\left[1-\left[\frac{p \mu_{h}}{\mu_{2}\left[1-\frac{\mathrm{n} 1 \mathrm{c}}{\mathrm{n} 1 \mathrm{c}+2\left(\theta+\theta_{1}\right)}\right]+\mu_{h}}+\frac{(1-p) \mu_{l}}{\mu_{2}\left[1-\frac{\mathrm{n} 1 \mathrm{c}}{\mathrm{n} 1 \mathrm{c}+2\left(\theta+\theta_{1}\right)}\right]+\mu_{l}}\right]\left\{\frac{m 1 ! c m 1}{\delta\left(c, 2\left(\theta+\theta_{1}\right)\right)}\right\}\right]\right\}} \\
& E_{36}=\frac{1-\frac{p \mu_{h}}{\mu_{2}\left[1-\frac{\mathrm{n} 1 \mathrm{c}}{\mathrm{n} 1 \mathrm{c}+\left(\theta+\theta_{1}+\theta_{2}\right)}\right]+\mu_{h}}-\frac{(1-p) \mu_{l}}{\mu_{2}\left[1-\frac{\mathrm{n} 1 \mathrm{c}}{\mathrm{n} 1 \mathrm{c}+\left(\theta+\theta_{1}+\theta_{2}\right)}\right]+\mu_{l}}}{\left\{\mu_{2}\left[1-\frac{\mathrm{n} 1 \mathrm{c}}{\mathrm{n} 1 \mathrm{c}+\left(\theta+\theta_{1}+\theta_{2}\right)}\right]\right\}\left\{\left[1-\left[\frac{p \mu_{h}}{\mu_{2}\left[1-\frac{\mathrm{n} 1 \mathrm{c}}{\mathrm{n} 1 \mathrm{c}+\left(\theta+\theta_{1}+\theta_{2}\right)}\right]+\mu_{h}}+\frac{(1-p) \mu_{l}}{\mu_{2}\left[1-\frac{\mathrm{n} 1 \mathrm{c}}{\mathrm{n} 1 \mathrm{c}+\left(\theta+\theta_{1}+\theta_{2}\right)}\right]+\mu_{l}}\right]\left\{\frac{m 1 ! c^{m 1}}{\delta\left(c,\left(\theta+\theta_{1}+\theta_{2}\right)\right)}\right\}\right]\right\}} \\
& E_{37}=\frac{1-\frac{p \mu_{h}}{\mu_{2}\left[1-\frac{\mathrm{n} 1 \mathrm{c}}{\mathrm{n} 1 \mathrm{c}+\left(2 \theta_{2}\right)}\right]+\mu_{h}}-\frac{(1-p) \mu_{l}}{\mu_{2}\left[1-\frac{\mathrm{n} 1 \mathrm{c}}{\mathrm{n} 1 \mathrm{c}+\left(2 \theta_{2}\right)}\right]+\mu_{l}}}{\left\{\mu_{2}\left[1-\frac{\mathrm{n} 1 \mathrm{c}}{\mathrm{n} 1 \mathrm{c}+\left(2 \theta_{2}\right)}\right]\right\}\left\{\left[1-\left[\frac{p \mu_{h}}{\mu_{2}\left[1-\frac{\mathrm{n} 1 \mathrm{c}}{\mathrm{n} 1 \mathrm{c}+\left(2 \theta_{2}\right)}\right]+\mu_{h}}+\frac{(1-p) \mu_{l}}{\mu_{2}\left[1-\frac{\mathrm{n} 1 \mathrm{c}}{\mathrm{n} 1 \mathrm{c}+\left(2 \theta_{2}\right)}\right]+\mu_{l}}\right]\left\{\frac{m 1 ! c m 1}{\delta\left(c,\left(2 \theta_{2}\right)\right)}\right\}\right]\right\}} \\
& 1-\frac{p \mu_{h}}{\mu_{2}\left[1-\frac{\mathrm{n} 1 \mathrm{c}}{\mathrm{n} 1 \mathrm{c}+\left(\theta+\theta_{1}\right)}\right]+\mu_{h}}-\frac{(1-p) \mu_{l}}{\mu_{2}\left[1-\frac{\mathrm{n} 1 \mathrm{c}}{\mathrm{n} 1 \mathrm{c}+\left(\theta+\theta_{1}\right)}\right]+\mu_{l}} \\
& E_{38}=\frac{\mathrm{n} 1 \mathrm{c}}{\left\{\mu_{2}\left[1-\frac{\mathrm{n} 1 \mathrm{c}+\left(\theta+\theta_{1}\right)}{\mathrm{n}}\right]\right\}\left\{\left[1-\left[\frac{p \mu_{h}}{\mu_{2}\left[1-\frac{\mathrm{n} 1 \mathrm{c}}{\mathrm{n} 1 \mathrm{c}+\left(\theta+\theta_{1}\right)}\right]+\mu_{h}}+\frac{(1-p) \mu_{l}}{\mu_{2}\left[1-\frac{\mathrm{n} 1 \mathrm{c}}{\mathrm{n} 1 \mathrm{c}+\left(\theta+\theta_{1}\right)}\right]+\mu_{l}}\right]\left\{\frac{m 1 ! c^{m 1}}{\delta\left(c,\left(\theta+\theta_{1}\right)\right)}\right\}\right]\right\}} \\
& E_{39}=\frac{1-\frac{p \mu_{h}}{\mu_{2}\left[1-\frac{\mathrm{n} 1 \mathrm{c}}{\mathrm{n} 1 \mathrm{c}+\left(\theta_{2}\right)}\right]+\mu_{h}}-\frac{(1-p) \mu_{l}}{\mu_{2}\left[1-\frac{\mathrm{n} 1 \mathrm{c}}{\mathrm{n} 1 \mathrm{c}+\left(\theta_{2}\right)}\right]+\mu_{l}}}{\left\{\mu_{2}\left[1-\frac{\mathrm{n} 1 \mathrm{c}}{\mathrm{n} 1 \mathrm{c}+\left(\theta_{2}\right)}\right]\right\}\left\{\left[1-\left[\frac{p \mu_{h}}{\mu_{2}\left[1-\frac{\mathrm{n} 1 \mathrm{c}}{\mathrm{n} 1 \mathrm{c}+\left(\theta_{2}\right)}\right]+\mu_{h}}+\frac{(1-p) \mu_{l}}{\mu_{2}\left[1-\frac{\mathrm{n} 1 \mathrm{c}}{\mathrm{n} 1 \mathrm{c}+\left(\theta_{2}\right)}\right]+\mu_{l}}\right]\left\{\frac{m 1 ! c m 1}{\delta\left(c,\left(\theta_{2}\right)\right)}\right\}\right]\right\}}
\end{aligned}
$$

\section{Case (iv)}

Sub Case (i) $n=3, r=1$

The mean time to recruitment is given by,

$$
\begin{aligned}
E(T)=\left\{\left\{\left\{p^{3} E_{41}\right.\right.\right. & \left.\left.+3 p^{2} q^{1} E_{42}+3 p^{1} q^{2} E_{43}+q^{3} E_{44}\right\}\right\}-3\left\{\left\{p^{2} E_{45}+2 p q E_{46}+q^{2} E_{47}\right\}\right\} \\
& \left.+3\left\{\left\{p^{1} E_{48}+q^{1} E_{49}\right\}\right\}\right\}
\end{aligned}
$$

Sub Case (ii) $n=3, r=2$

The mean time to recruitment is given by

$$
E(T)=\left\{\left\{3\left\{p^{3} E_{41}+3 p^{2} q^{1} E_{42}+3 p^{1} q^{2} E_{43}+q^{3} E_{44}\right\}\right\}-2\left\{p^{2} E_{45}+2 p q E_{46}+q^{2} E_{47}\right\}\right\}
$$

\section{Sub Case (iii) $n=3, r=3$}

The mean time to recruitment is given by

where

$$
E(T)=\left\{p^{3} E_{41}+3 p^{2} q^{1} E_{42}+3 p^{1} q^{2} E_{43}+q^{3} E_{44}\right\}
$$

$$
\begin{aligned}
& E_{41}=\frac{1-\frac{p \mu_{h}}{\mu_{2}\left[1-\frac{n 1 ! c^{n 1}}{\delta\left(c, 3\left(\theta+\theta_{1}\right)\right)}\right]+\mu_{h}}-\frac{(1-p) \mu_{l}}{\mu_{2}\left[1-\frac{n 1 ! c^{n 1}}{\delta\left(c, 3\left(\theta+\theta_{1}\right)\right)}\right]+\mu_{l}}}{\left\{\mu_{2}\left[1-\frac{n 1 ! c^{n 1}}{\delta\left(c, 3\left(\theta+\theta_{1}\right)\right)}\right]\right\}\left\{\left[1-\left[\frac{p \mu_{h}}{\mu_{2}\left[1-\frac{n 1 ! c^{n 1}}{\delta\left(c, 3\left(\theta+\theta_{1}\right)\right)}\right]+\mu_{h}}+\frac{(1-p) \mu_{l}}{\mu_{2}\left[1-\frac{n 1 ! c^{n 1}}{\delta\left(c, 3\left(\theta+\theta_{1}\right)\right)}\right]+\mu_{l}}\right]\left\{\frac{m 1 ! c^{m 1}}{\delta\left(c, 3\left(\theta+\theta_{1}\right)\right)}\right\}\right]\right\}} \\
& E_{42}=\frac{1-\frac{n \mu_{h}}{\mu_{2}\left[1-\frac{n 1 c^{n 1}}{\left.\delta\left(c, 2 \theta+c_{1}+\theta_{2}\right)\right)}\right]+\mu_{h}}-\frac{(1-p) \mu_{l}}{\mu_{2}\left[1-\frac{n 1 ! c^{n 1}}{\delta\left(c,\left(2 \theta+2 \theta_{1}+\theta_{2}\right)\right)}\right]+\mu_{l}}}{\left.\left\{\mu_{2}\left[1-\frac{n 1 ! c^{n 1}}{\delta\left(c,\left(2 \theta+2 \theta_{1}+\theta_{2}\right)\right)}\right]\right\}\left\{1-\left[\frac{n \mu_{h}}{\mu_{2}\left[1-\frac{n ! c^{n 1}}{\delta\left(c,\left(2 \theta+2 \theta_{1}+\theta_{2}\right)\right)}\right]+\mu_{h}}+\frac{(1-p) \mu_{l}}{\mu_{2}\left[1-\frac{n 1 ! c}{\left.\delta\left(c, 2 \theta+2 \theta_{1}+\theta_{2}\right)\right)}\right]+\mu_{l}}\right]\left\{\frac{m 1 ! c^{m 1}}{\delta\left(c,\left(2 \theta+2 \theta_{1}+\theta_{2}\right)\right)}\right\}\right]\right\}}
\end{aligned}
$$


Mean Time to Recruitment for a Multigrade Manpower System with Two Sources ...

$$
\begin{aligned}
& E_{43}=\frac{1-\frac{p \mu_{h}}{\mu_{2}\left[1-\frac{n 1 ! c^{n 1}}{\delta\left(c,\left(\theta+\theta_{1}+2 \theta_{2}\right)\right)}\right]+\mu_{h}}-\frac{(1-p) \mu_{l}}{\mu_{2}\left[1-\frac{n ! c^{n 1}}{\delta\left(c,\left(\theta+\theta_{1}+2 \theta_{2}\right)\right)}\right]+\mu_{l}}}{\left.\left\{\mu_{2}\left[1-\frac{n 1 ! c^{n 1}}{\delta\left(c,\left(\theta+\theta_{1}+2 \theta_{2}\right)\right)}\right]\right\}\left\{1-\left[\frac{n \mu_{h}}{\mu_{2}\left[1-\frac{n 1 ! c^{n 1}}{\delta\left(c,\left(\theta+\theta_{1}+2 \theta_{2}\right)\right)}\right]+\mu_{h}}+\frac{(1-p) \mu_{l}}{\mu_{2}\left[1-\frac{n 1 ! c^{n 1}}{\delta\left(c,\left(\theta+\theta_{1}+2 \theta_{2}\right)\right)}\right]+\mu_{l}}\right]\left\{\frac{\mathrm{m} 1 \mathrm{c}}{\mathrm{m} 1 \mathrm{c}+\left(\theta+\theta_{1}+2 \theta_{2}\right)}\right\}\right]\right\}} \\
& 1-\frac{p \mu_{h}}{\mu_{2}\left[1-\frac{n 1 ! c^{n 1}}{\delta\left(c, 3\left(\theta_{2}\right)\right)}\right]+\mu_{h}}-\frac{(1-p) \mu_{l}}{\mu_{2}\left[1-\frac{n 1 ! c^{n 1}}{\delta\left(c, 3\left(\theta_{2}\right)\right)}\right]+\mu_{l}} \\
& E_{44}=\frac{\mu_{2}\left[1-\frac{n\left(c, 3\left(\theta_{2}\right)\right)}{\delta\left(\mu_{h}\right.} \mu_{2}\left[1-\frac{n\left(c, 3\left(\theta_{2}\right)\right)}{\delta\left(\mu_{l}\right.}\right.\right.}{\left\{\mu_{2}\left[1-\frac{n 1 ! c^{n 1}}{\delta\left(c, 3\left(\theta_{2}\right)\right)}\right]\right\}\left\{\left[1-\left[\frac{p \mu_{h}}{\mu_{2}\left[1-\frac{n 1 ! c^{n 1}}{\delta\left(c, 3\left(\theta_{2}\right)\right)}\right]+\mu_{h}}+\frac{(1-p) \mu_{l}}{\mu_{2}\left[1-\frac{n 1 ! c^{11}}{\delta\left(c, 3\left(\theta_{2}\right)\right)}\right]+\mu_{l}}\right]\left\{\frac{m 1 ! c^{m 1}}{\delta\left(c, 3\left(\theta_{2}\right)\right)}\right\}\right]\right\}} \\
& 1-\frac{p \mu_{h}}{\mu_{2}\left[1-\frac{n 1 ! c^{n 1}}{\delta\left(c, 2\left(\theta+\theta_{1}\right)\right)}\right]+\mu_{h}}-\frac{(1-p) \mu_{l}}{\mu_{2}\left[1-\frac{n 1 ! c^{n 1}}{\delta\left(c, 2\left(\theta+\theta_{1}\right)\right)}\right]+\mu_{l}} \\
& E_{45}=\frac{\left\{\mu_{2}\left[1-\frac{n 1 ! c^{n 1}}{\delta\left(c, 2\left(\theta+\theta_{1}\right)\right)}\right]\right\}\left\{\left[1-\left[\frac{p \mu_{h}}{\mu_{2}\left[1-\frac{n 1 ! c^{n 1}}{\delta\left(c, 2\left(\theta+\theta_{1}\right)\right.}\right]+\mu_{h}}+\frac{(1-p) \mu_{l}}{\mu_{2}\left[1-\frac{n 1 ! c^{n 1}}{\delta\left(c, 2\left(\theta+\theta_{1}\right)\right)}\right]+\mu_{l}}\right]\left\{\frac{m 1 ! c^{m 1}}{\delta\left(c, 2\left(\theta+\theta_{1}\right)\right)}\right\}\right]\right\}}{\delta\left(c, 2\left(\theta+\theta_{1}\right)\right)} \\
& 1-\frac{p \mu_{h}}{\mu_{2}\left[1-\frac{n 1 ! c^{n 1}}{\delta\left(c,\left(\theta+\theta_{1}+\theta_{2}\right)\right)}\right]+\mu_{h}}-\frac{(1-p) \mu_{l}}{\mu_{2}\left[1-\frac{n 1 ! c^{n 1}}{\delta\left(c,\left(\theta+\theta_{1}+\theta_{2}\right)\right)}\right]+\mu_{l}} \\
& E_{46}=\frac{\mu_{2}\left[1-\frac{\left.n\left(c, \theta+\theta_{1}+\theta_{2}\right)\right)}{\delta}+\mu_{h}\right.}{\left\{\mu_{2}\left[1-\frac{n 1 ! c^{n 1}}{\delta\left(c,\left(\theta+\theta_{1}+\theta_{2}\right)\right)}\right]\right\}\left\{\left[1-\left[\frac{p \mu_{h}}{\mu_{2}\left[1-\frac{n 1 ! c^{n 1}}{\delta\left(c,\left(\theta+\theta_{1}+\theta_{2}\right)\right.}\right]+\mu_{h}}+\frac{(1-p) \mu_{l}}{\mu_{2}\left[1-\frac{n ! c c^{\prime}}{\delta\left(c,\left(\theta+\theta_{1}+\theta_{2}\right)\right)}\right]+\mu_{l}}\right]\left\{\frac{m 1 ! c^{m 1}}{\delta\left(c,\left(\theta+\theta_{1}+\theta_{2}\right)\right)}\right\}\right]\right\}} \\
& 1-\frac{p \mu_{h}}{\mu_{2}\left[1-\frac{n 1 ! c^{n 1}}{\delta\left(c, 2\left(\theta_{2}\right)\right)}\right]+\mu_{h}}-\frac{(1-p) \mu_{l}}{\mu_{2}\left[1-\frac{n 1 ! c^{n 1}}{\delta\left(c, 2\left(\theta_{2}\right)\right)}\right]+\mu_{l}} \\
& E_{47}=\frac{\left.\mu_{2}\left[1-\frac{n 1 ! c^{n 1}}{\delta\left(c, 2\left(\theta_{2}\right)\right)}\right]\right\}\left\{\left[1-\left[\frac{p \mu_{h}}{\mu_{2}\left[1-\frac{n 1 ! c^{n 1}}{\delta\left(c, 2\left(\theta_{2}\right)\right)}\right]+\mu_{h}}+\frac{(1-p) \mu_{l}}{\mu_{2}\left[1-\frac{n 1 ! c^{n 1}}{\delta\left(c, 2\left(\theta_{2}\right)\right)}\right]+\mu_{l}}\right]\left\{\frac{m 1 ! c^{m 1}}{\delta\left(c, 2\left(\theta_{2}\right)\right)}\right\}\right]\right\}}{\delta(c, p) \mu_{l}} \\
& 1-\frac{p \mu_{h}}{\mu_{2}\left[1-\frac{n 1 ! c^{n 1}}{\delta\left(c,\left(\theta+\theta_{1}\right)\right)}\right]+\mu_{h}}-\frac{(1-p) \mu_{l}}{\mu_{2}\left[1-\frac{n 1 ! c^{n 1}}{\delta\left(c,\left(\theta+\theta_{1}\right)\right)}\right]+\mu_{l}} \\
& E_{48}=\frac{\mu_{2}\left[1-\frac{1\left(c,\left(\theta+\theta_{1}\right)\right)}{\delta\left(\mu_{h}\right.} \mu_{2}\left[1-\frac{n\left(c,\left(\theta+\theta_{1}\right)\right)}{\delta\left(\mu_{l}\right.}\right.\right.}{\left\{\mu_{2}\left[1-\frac{n 1 ! c^{n 1}}{\delta\left(c,\left(\theta+\theta_{1}\right)\right)}\right]\right\}\left\{\left[1-\left[\frac{p \mu_{h}}{\mu_{2}\left[1-\frac{n ! c^{n 1}}{\delta\left(c,\left(\theta+\theta_{1}\right)\right.}\right]+\mu_{h}}+\frac{(1-p) \mu_{l}}{\mu_{2}\left[1-\frac{n 1 ! c^{n 1}}{\delta\left(c,\left(\theta+\theta_{1}\right)\right.}\right]+\mu_{l}}\right]\left\{\frac{m 1 ! c^{m 1}}{\delta\left(c,\left(\theta+\theta_{1}\right)\right)}\right\}\right]\right\}} \\
& 1-\frac{p \mu_{h}}{\mu_{2}\left[1-\frac{n 1 ! c^{n 1}}{\delta\left(c,\left(\theta_{2}\right)\right)}\right]+\mu_{h}}-\frac{(1-p) \mu_{l}}{\mu_{2}\left[1-\frac{n 1 ! c^{n 1}}{\delta\left(c,\left(\theta_{2}\right)\right)}\right]+\mu_{l}}
\end{aligned}
$$

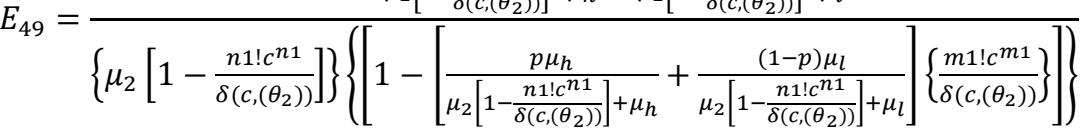

\section{Comparison table}

The influence of parameters on the performance measures namely the mean time for recruitment is studied numerically. In the following tables these performance measures are calculated by varying the parameter ' $\lambda_{1}{ }^{\prime},{ }^{\prime} \lambda_{2}{ }^{\prime}, \mu_{h}{ }^{\prime},{ }^{\prime} \mu_{l}{ }^{\prime},{ }^{\prime} \mu_{2}{ }^{\prime},{ }^{\prime} \theta_{1}{ }^{\prime}$ and ${ }^{\prime} \theta_{2}{ }^{\prime}$ one at a time and taking the parameters $p=0.3,1-p=q=0.7 \theta=0.2$ 
K.Srividhya and S.Sendhamizh Selvi

\begin{tabular}{cccccclll}
$\begin{array}{c}\text { Case (i) } \\
\mathbf{C}\end{array}$ & $\boldsymbol{\mu}_{\boldsymbol{h}}$ & $\boldsymbol{\mu}_{\boldsymbol{l}}$ & $\boldsymbol{\mu}_{\mathbf{2}}$ & $\boldsymbol{\theta}_{\mathbf{1}}$ & $\boldsymbol{\theta}_{\mathbf{2}}$ & $\begin{array}{l}\text { SubCase(i) } \\
\mathbf{E ( T )}\end{array}$ & $\begin{array}{l}\text { SubCase(ii) } \\
\mathbf{E ( T )}\end{array}$ & $\begin{array}{l}\text { SubCase(iii) } \\
\mathbf{E ( T )}\end{array}$ \\
0.2 & 0.4 & 0.2 & 0.5 & 0.3 & 0.4 & 5.4177 & 3.1946 & 2.0826 \\
0.2 & 0.4 & 0.2 & 0.5 & 0.3 & 0.5 & 4.8055 & 2.9265 & 1.9766 \\
0.2 & 0.4 & 0.2 & 0.5 & 0.3 & 0.6 & 4.4110 & 2.7393 & 1.8998 \\
\hline 0.2 & 0.4 & 0.2 & 0.5 & 0.4 & 0.5 & 4.6400 & 2.8443 & 1.9421 \\
0.2 & 0.4 & 0.2 & 0.5 & 0.5 & 0.5 & 4.5305 & 2.7805 & 1.9134 \\
0.2 & 0.4 & 0.2 & 0.5 & 0.6 & 0.5 & 4.4542 & 2.7296 & 1.8890 \\
0.2 & 0.4 & 0.2 & 0.6 & 0.3 & 0.5 & 4.1744 & 2.5504 & 1.7301 \\
0.2 & 0.4 & 0.2 & 0.7 & 0.3 & 0.5 & 3.6902 & 2.2602 & 1.5385 \\
0.2 & 0.4 & 0.2 & 0.8 & 0.3 & 0.5 & 3.3068 & 2.0295 & 1.3852 \\
0.2 & 0.4 & 0.3 & 0.6 & 0.3 & 0.5 & 3.8908 & 2.3701 & 1.6000 \\
0.2 & 0.4 & 0.4 & 0.6 & 0.3 & 0.5 & 3.6664 & 2.2259 & 1.4957 \\
0.2 & 0.4 & 0.5 & 0.6 & 0.3 & 0.5 & 3.4845 & 2.1081 & 1.4102 \\
0.2 & 0.5 & 0.2 & 0.6 & 0.3 & 0.5 & 4.1212 & 2.5106 & 1.6977 \\
0.2 & 0.6 & 0.2 & 0.6 & 0.3 & 0.5 & 4.0800 & 2.4789 & 1.6712 \\
0.2 & 0.7 & 0.2 & 0.6 & 0.3 & 0.5 & 4.0473 & 2.4530 & 1.6491 \\
0.5 & 0.4 & 0.2 & 0.6 & 0.3 & 0.5 & 8.6245 & 4.5783 & 2.5473 \\
0.6 & 0.4 & 0.2 & 0.6 & 0.3 & 0.5 & 10.1078 & 5.2528 & 2.8183 \\
0.7 & 0.4 & 0.2 & 0.6 & 0.3 & 0.5 & 11.5912 & 5.9272 & 3.0890
\end{tabular}

Case (ii)

\begin{tabular}{rrrrrrlll}
$\mathbf{c}$ & $\boldsymbol{\mu}_{\boldsymbol{h}}$ & $\boldsymbol{\mu}_{\boldsymbol{l}}$ & $\boldsymbol{\mu}_{\mathbf{2}}$ & $\boldsymbol{\theta}_{\mathbf{1}}$ & $\boldsymbol{\theta}_{\mathbf{2}}$ & $\begin{array}{l}\text { SubCase(i) } \\
\mathbf{E ( T )}\end{array}$ & $\begin{array}{l}\text { SubCase(ii) } \\
\mathbf{E ( T )}\end{array}$ & $\begin{array}{l}\text { SubCase(iii) } \\
\mathbf{E}(\mathbf{T})\end{array}$ \\
0.2 & 0.4 & 0.2 & 0.5 & 0.3 & 0.4 & 2.2688 & 1.8299 & 1.5735 \\
0.2 & 0.4 & 0.2 & 0.5 & 0.3 & 0.5 & 2.1458 & 1.7761 & 1.5469 \\
0.2 & 0.4 & 0.2 & 0.5 & 0.3 & 0.6 & 2.0698 & 1.7373 & 1.5266 \\
\hline 0.2 & 0.4 & 0.2 & 0.5 & 0.4 & 0.5 & 2.1139 & 1.7591 & 1.5379 \\
0.2 & 0.4 & 0.2 & 0.5 & 0.5 & 0.5 & 2.0930 & 1.7456 & 1.5301 \\
0.2 & 0.4 & 0.2 & 0.5 & 0.6 & 0.5 & 2.0785 & 1.7346 & 1.5234 \\
0.2 & 0.4 & 0.2 & 0.6 & 0.3 & 0.5 & 1.8156 & 1.5175 & 1.3398 \\
0.2 & 0.4 & 0.2 & 0.7 & 0.3 & 0.5 & 1.5233 & 1.3246 & 1.1818 \\
0.2 & 0.4 & 0.2 & 0.8 & 0.3 & 0.5 & 1.3879 & 1.1751 & 1.0572 \\
0.2 & 0.4 & 0.3 & 0.6 & 0.3 & 0.5 & 2.0835 & 1.6952 & 1.4430 \\
0.2 & 0.4 & 0.4 & 0.6 & 0.3 & 0.5 & 2.0276 & 1.6254 & 1.3578 \\
0.2 & 0.4 & 0.5 & 0.6 & 0.3 & 0.5 & 1.9775 & 1.5647 & 1.2868 \\
0.2 & 0.5 & 0.2 & 0.6 & 0.3 & 0.5 & 1.9855 & 1.5021 & 1.3184 \\
0.2 & 0.6 & 0.2 & 0.6 & 0.3 & 0.5 & 1.9842 & 1.4888 & 1.3004 \\
0.2 & 0.7 & 0.2 & 0.6 & 0.3 & 0.5 & 1.9803 & 1.4774 & 1.2849 \\
0.5 & 0.4 & 0.2 & 0.6 & 0.3 & 0.5 & 4.9608 & 3.0336 & 2.2945 \\
0.6 & 0.4 & 0.2 & 0.6 & 0.3 & 0.5 & 5.6478 & 3.3125 & 2.3908 \\
0.7 & 0.4 & 0.2 & 0.6 & 0.3 & 0.5 & 6.4873 & 3.6072 & 2.4900
\end{tabular}


Mean Time to Recruitment for a Multigrade Manpower System with Two Sources ...

Case (iii)

\begin{tabular}{rccccclll}
$\boldsymbol{c}$ & $\boldsymbol{\mu}_{\boldsymbol{h}}$ & $\boldsymbol{\mu}_{\boldsymbol{l}}$ & $\boldsymbol{\mu}_{\mathbf{2}}$ & $\boldsymbol{\theta}_{\mathbf{1}}$ & $\boldsymbol{\theta}_{\mathbf{2}}$ & $\begin{array}{l}\text { SubCase(i) } \\
\mathbf{E ( T )}\end{array}$ & $\begin{array}{l}\text { SubCase(ii) } \\
\mathbf{E ( T )}\end{array}$ & $\begin{array}{l}\text { SubCase(iii) } \\
\mathbf{E}(\mathbf{T})\end{array}$ \\
0.2 & 0.4 & 0.2 & 0.5 & 0.3 & 0.4 & 2.9208 & 2.1645 & 1.7002 \\
0.2 & 0.4 & 0.2 & 0.5 & 0.3 & 0.5 & 2.7279 & 2.0635 & 1.6533 \\
0.2 & 0.4 & 0.2 & 0.5 & 0.3 & 0.6 & 2.5991 & 1.9898 & 1.6182 \\
\hline 0.2 & 0.4 & 0.2 & 0.5 & 0.4 & 0.5 & 2.6740 & 2.0313 & 1.6376 \\
0.2 & 0.4 & 0.2 & 0.5 & 0.5 & 0.5 & 2.6371 & 2.0057 & 1.6244 \\
0.2 & 0.4 & 0.2 & 0.5 & 0.6 & 0.5 & 2.6106 & 1.9847 & 1.6130 \\
0.2 & 0.4 & 0.2 & 0.6 & 0.3 & 0.5 & 2.5143 & 1.8686 & 1.4762 \\
0.2 & 0.4 & 0.2 & 0.7 & 0.3 & 0.5 & 2.3313 & 1.7075 & 1.3338 \\
0.2 & 0.4 & 0.2 & 0.8 & 0.3 & 0.5 & 2.1719 & 1.5721 & 1.2166 \\
0.2 & 0.4 & 0.3 & 0.6 & 0.3 & 0.5 & 2.2707 & 1.7770 & 1.4621 \\
0.2 & 0.4 & 0.4 & 0.6 & 0.3 & 0.5 & 1.9724 & 1.5770 & 1.3214 \\
0.2 & 0.4 & 0.5 & 0.6 & 0.3 & 0.5 & 1.7631 & 1.4298 & 1.2136 \\
0.2 & 0.5 & 0.2 & 0.6 & 0.3 & 0.5 & 2.6395 & 2.0006 & 1.6071 \\
0.2 & 0.6 & 0.2 & 0.6 & 0.3 & 0.5 & 2.5744 & 1.9523 & 1.5705 \\
0.2 & 0.7 & 0.2 & 0.6 & 0.3 & 0.5 & 2.5245 & 1.9142 & 1.5409 \\
0.5 & 0.4 & 0.2 & 0.6 & 0.3 & 0.5 & 4.2888 & 2.8613 & 2.0351 \\
0.6 & 0.4 & 0.2 & 0.6 & 0.3 & 0.5 & 4.8017 & 3.1036 & 2.1482 \\
0.7 & 0.4 & 0.2 & 0.6 & 0.3 & 0.5 & 5.3160 & 3.3420 & 2.2572
\end{tabular}

Case (iv)

\begin{tabular}{ccccccccc}
$\boldsymbol{c}$ & $\boldsymbol{\mu}_{\boldsymbol{h}}$ & $\boldsymbol{\mu}_{\boldsymbol{l}}$ & $\boldsymbol{\mu}_{\mathbf{2}}$ & $\boldsymbol{\theta}_{\mathbf{1}}$ & $\boldsymbol{\theta}_{\mathbf{2}}$ & $\begin{array}{l}\text { SubCase(i) } \\
\mathrm{E}(\mathrm{T})\end{array}$ & $\begin{array}{l}\text { Case(ii) } \\
\mathbf{E ( T )}\end{array}$ & $\begin{array}{l}\text { Case(iii) } \\
\mathbf{E}(\mathbf{T})\end{array}$ \\
0.2 & 0.4 & 0.2 & 0.5 & 0.3 & 0.4 & 1.5077 & 1.3694 & 1.3353 \\
0.2 & 0.4 & 0.2 & 0.5 & 0.3 & 0.5 & 1.4978 & 1.3662 & 1.3387 \\
0.2 & 0.4 & 0.2 & 0.5 & 0.3 & 0.6 & 1.4915 & 1.3640 & 1.3382 \\
\hline 0.2 & 0.4 & 0.2 & 0.5 & 0.4 & 0.5 & 1.5537 & 1.3808 & 1.3414 \\
0.2 & 0.4 & 0.2 & 0.5 & 0.5 & 0.5 & 1.5242 & 1.3739 & 1.3402 \\
0.2 & 0.4 & 0.2 & 0.5 & 0.6 & 0.5 & 1.5077 & 1.3694 & 1.3393 \\
0.2 & 0.4 & 0.2 & 0.6 & 0.3 & 0.5 & 1.3475 & 1.2120 & 1.1813 \\
0.2 & 0.4 & 0.2 & 0.7 & 0.3 & 0.5 & 1.2077 & 1.0845 & 1.0563 \\
0.2 & 0.4 & 0.2 & 0.8 & 0.3 & 0.5 & 1.0944 & 0.9815 & 0.9554 \\
0.2 & 0.4 & 0.3 & 0.6 & 0.3 & 0.5 & 1.3723 & 1.2425 & 1.2140 \\
0.2 & 0.4 & 0.4 & 0.6 & 0.3 & 0.5 & 1.2561 & 1.1408 & 1.1160 \\
0.2 & 0.4 & 0.5 & 0.6 & 0.3 & 0.5 & 1.1644 & 1.0597 & 1.0376 \\
0.2 & 0.5 & 0.2 & 0.6 & 0.3 & 0.5 & 1.3454 & 1.1836 & 1.1538 \\
0.2 & 0.6 & 0.2 & 0.6 & 0.3 & 0.5 & 1.3185 & 1.1600 & 1.1309 \\
0.2 & 0.7 & 0.2 & 0.6 & 0.3 & 0.5 & 1.2960 & 1.1401 & 1.1116 \\
0.5 & 0.4 & 0.2 & 0.6 & 0.3 & 0.5 & 3.4926 & 2.2547 & 1.8665 \\
0.6 & 0.4 & 0.2 & 0.6 & 0.3 & 0.5 & 4.0424 & 2.4384 & 1.9139 \\
0.7 & 0.4 & 0.2 & 0.6 & 0.3 & 0.5 & 4.6298 & 2.6370 & 1.9673
\end{tabular}


K.Srividhya and S.Sendhamizh Selvi

Findings:

- As 'c' the loss of manpower increases, (i.e) the mean loss of man power decreases, and hence time taken for threshold crossing (i.e) the mean time to recruitment increases.

- As ' $\mu_{h}{ }^{\prime},{ }^{\prime} \mu_{l}{ }^{\prime}$ and ' $\mu_{2}{ }^{\prime}$ the parameter for policy and transfer decision increases (i.e) mean policy and transfer decision time decreases, and hence the time taken for threshold crossing(i.e) the mean time to recruitment decreases.

- As ' $\theta_{1}{ }^{\prime}$ and ${ }^{\prime} \theta_{2}$ 'the the mean threshold level decreases and hence the time taken for threshold crossing i.e., mean time to recruitment decreases

\section{Conclusions}

In the context of providing scope for future work, it is worthwhile to mention that the present work can be studied by considering different types of loss in manpower also.

\section{REFERENCES}

1. D.J.Barthlomew and A.F.Forbes, Statistical techniques for manpower planning, John Wiley \& Sons (1979).

2. R.C.Grinold and K.J.Marshall, Man Power Planning, North Holland, Newyork (1977).

3. J.Sridharan, K.Parameswari and A.A.Srinivasan, A stochastic model on time to recruitment in a two grademanpower system based on order statistics, InternationalJournal of Mathematical Sciences and Engineering Applications, 6(5) (2012) 23-30.

4. J.Sridharan, K.Parameswari and A.Srinivasan, A stochastic model on time to recruitment in a two grademanpower system involving extended exponential threshold based on order statistics, Bessel Journal of Mathematics, 3(1) (2013):39-49.

5. J.Sridharan, K.Parameswari and A.Srinivasan, A stochastic model on time to recruitment in a two grade manpower system involving extended exponential and exponential threshold based on order statistics, Archimedes Journal of Mathematics, 3(1) (2013) 41-50.

6. J.Sridharan, K.Parameswari and A.Srinivasan, A stochastic model on time to recruitment in a two grademanpower system basedon order statistics when thethreshold distribution having SCBZ property, CayleyJournal of Mathematics, 1(2) (2012 ) 101-112

7. J.Sridharan, K.Parameswari and A.Srinivasan, Time torecruitment in a two grade manpower system based on order statistics, Antarctica Journal of Mathematics, 10(2) (2013 ) 169-181.

8. K.Parameswari and A.Srinivasan, Estimation of variance of time to recruitment for a two grade manpower systemwith two types of decisions when the wastages form ageometric process, International Journal of Mathematics Trends and Technology, 33(3) (2016).

9. S.Dhivya, V.Vasudevan and A.Srinivasn, Stochastic models for the time to recruitment in a two grademanpower system using same geometric process for theinter decision times, proceedings of mathematical andcomputational models, PSG college of technology (ICMCM), Narosa publishing House, pp.276-283, Dec -2011 
Mean Time to Recruitment for a Multigrade Manpower System with Two Sources ...

10. S.Vidhya, A study on some stochastic models for amulti graded manpower system, $\mathrm{Ph} . \mathrm{D}$ thesis, Bharathidasan University (2011).

11. K.Srividhya, S.Sendhamizhi Selvi, Mean time to recruitment for a multi grade manpower system with single threshold, single source of depletion when interpolicy decisions form an order statistics, IOSR Journal of Mathematics, 13(3) (2012) 33-38.

12. K.Srividhya, S.Sendhamizhselvi, Mean time to recruitment for a multi grade man power system with single threshold, single source of depletion when inter policy decisions form a geometric process, International Journal of Mathematics Trends and Technology, 45(1) (2017) 11-15. 